\title{
Synthesis, Molecular Modeling, and Biological Evaluation of Novel Benzimidazole Derivatives as Inhibitors of Hepatitis C Virus RNA Replication
}

\author{
Hoda Ibrahim El Diwani, ${ }^{a}$ Heba Tawfik Abdel-Mohsen, ${ }^{a}$ Ismail Salama, ${ }^{*, b ;}$ \\ Fatma Abdel-Fattah Ragab, ${ }^{c}$ Mostafa Mahmoud Ramla, ${ }^{a}$ Shadia Ahmed Galal, ${ }^{a}$ \\ Mohamed Mostafa Abdalla, ${ }^{d}$ Abeer Abdel-Wahab, ${ }^{e}$ and Maha Adel El Demellawy ${ }^{e}$ \\ ${ }^{a}$ Department of Chemistry of Natural and Microbial Products, Division of Pharmaceutical and Drug Industries \\ Research, National Research Center; Cairo, Egypt: ${ }^{b}$ Department of Pharmaceutical Chemistry, Faculty of Pharmacy, \\ Suez Canal University; Ismailia, Egypt: ${ }^{c}$ Department of Pharmaceutical Chemistry, Faculty of Pharmacy, Cairo \\ University; Cairo, Egypt: ${ }^{d}$ Research Unit, Saco Pharm. Co.; 6th October City, Cairo, Egypt: and ${ }^{e}$ Medical \\ Biotechnology Department (MBD), Genetic Engineering \& Biotechnology Research Institute (GEBRI), City of \\ Scientific Research \& Technology Applications; Alexandria, Egypt. \\ Received December 31, 2013; accepted May 20, 2014
}

In this study, synthesis and docking studies of a series of new benzimidazole derivatives linked to substituted pyrimidines either through the methylenethio linkage or its bioisosteric methylene amino bridge were carried out. All the synthesized compounds were evaluated for their hepatitis C virus (HCV) RNA replication-inhibitory activity. Compounds $4 \mathrm{~d}, 4 \mathrm{f}$, and $4 \mathrm{~h}$ were found to be more potent than VX-950 $\left(\mathrm{IC}_{50 / 90}\right.$ of $4 \mathrm{~d}=0.123 / 0.321,4 \mathrm{f}=0.145 / 0.345,4 \mathrm{~h}=0.129 / 0.432, \mathrm{VX}-950=0.20 / 0.45 \mu \mathrm{M}$, respectively) and $6 \mathrm{~d}$ $\left(\mathrm{IC}_{50 / 90}=0.116 / 0.452 \mu \mathrm{M}\right)$ displayed activity very similar to that of the standard. Compounds $4 \mathrm{~d}, 4 \mathrm{f}, 4 \mathrm{~h}$, and $6 \mathrm{~d}$ were potent HCV RNA replication inhibitors and are good drug candidates for further investigations.

Key words synthesis; benzimidazole; pyrimidine; hepatitis C virus; viral RNA replication inhibitor

As estimated by WHO, 170 million persons worldwide are infected with hepatitis $\mathrm{C}$ virus (HCV) which causes chronic liver disease. $\mathrm{HCV}$ is a positive stranded RNA virus and is a member of the Flaviviridae family of viruses. ${ }^{1,2)}$ Combined therapy of $\mathrm{HCV}$ infections with pegylated alpha interferon (IFN- $\alpha$ ) and ribavirin is only effective in $50-60 \%$ of infected individuals and is associated with serious side effects such as depression, flu-like symptoms, fatigue and hemolytic anemia caused by ribavirin. ${ }^{3,4)}$

There is an unmet need for potent and selective inhibitors of HCV replication. Significant research efforts are currently directed towards targeting viral enzymes especially NS3-4A serine protease $\mathrm{e}^{5-8)}$ and NS5B RNA-dependent RNA polymerase which are both required for virus propagation. ${ }^{9-11)}$

The NS3-4A serine protease has been an attractive target in the development of new antivirals with activities against $\mathrm{HCV}$ since it is essential for viral replication. ${ }^{9-11)}$ The serine protease activity of NS3 in complex with the NS4A cofactor is responsible for the proteolytic cleavage at four junctions of the HCV polyprotein precursor: NS3-NS4A (self cleavage), NS4ANS4B, NS4B-NS5A, and NS5A-NS5B.,12-17)

Crystal structure of the serine protease domain of the NS3, with its essential cofactor NS4A, ${ }^{18,19)}$ revealed that the NS3-4A complex adopts a chymotrypsin/trypsin-like fold with structurally conserved regions typical of small chymotrypsin-like proteases. $^{20,21)}$ The N-terminal region of NS3-4A (residues 1-93 of NS3 and residues 21-34 of NS4A) contains an eightstranded $\beta$-barrel motif, with one of the strands contributed by the NS4A cofactor. ${ }^{20)}$ The C-terminal region (residues 94-175)

\footnotetext{
The authors declare no conflict of interest.

${ }^{\dagger}$ Present address: Department Chemistry and Pharmacy, Emil Fischer Center, Friedrich-Alexander University; Schuhstrasse 19, D-91052 Erlangen, Germany.
}

contains a six-stranded-barrel that ends with a helix. The active site (His: 57, Asp: 81, and Ser: 139) is located between these two regions and is formed by a shallow solvent exposed pocket to which the inhibitors bind in an extended conformation. ${ }^{18-21)}$

Sofosbuvir (GS-7977), a uridine nucleotide analogue, and an inhibitor of HCV NS5B polymerase is effective against HCV genotypes 1, 2, 3, 4, and 6. Sofosbuvir, especially in interferon-free combinations, represents a very promising option in the treatment of chronic hepatitis $\mathrm{C}^{22)}$

Currently, different anti-HCV treatments depend on the inhibition of $\mathrm{HCV}$ NS3-4A serine protease. Telaprevir (VX-950), boceprevir and BILN 2061 (Fig. 1) are examples of well known HCV NS3-4A serine protease inhibtors. ${ }^{23,24)}$

Telaprevir, a small molecule peptidomimetic, forms a covalent but reversible complex with the HCV NS3-4A serine protease in a slow process (slow-on). It was discovered to have excellent efficacy in the clinical setting. ${ }^{23-27)}$

The benzimidazole scaffold is common in different antiHCV agents with different modes of actions.

Derivatives of bis-benzimidazolemethane were discovered to be highly potent, reversible and selective serine protease inhibitors e.g., CRA-6336 ${ }^{28,29)}$ (Fig. 2).

Recently, we have synthesized a novel series of benzimidazole derivatives having a pyrimidothiomethylene group at the 2-position and it was found that it has a potent antitumor activity against different cell lines. ${ }^{30)}$ In this study we were interested in finding out whether replacing the 2-methylbenzimidzole moiety of bis-benzimidazolemethane CRA-6336 with 2-pyrimidomethylenethio moiety or its bioisosteric 2pyrimidomethyleneamino group would have a positive impact on the inhibitory activity against HCV. Hence, introducing new scaffolds for the development of wide range of antiviral agents. 
After the successful discovery of the new scaffold, we started to carry out different modifications and the corresponding inhibitory activity was examined. These modifications included the introduction of different substituents on the 4phenyl ring of the pyrimidine moiety, bioisosteric replacement of the phenyl ring with heterocyclic rings, and hydrolysis of the 5-cyano substituent on the pyrimidine nucleus to car-<smiles>CCC[C@H](NC(=O)[C@@H]1[C@H]2CCC[C@H]2CN1C(=O)[C@@H](NC(=O)[C@@H](NC(=O)c1cnccn1)C1CCCCC1)C(C)(C)C)C(=O)C(=O)NC1CC1</smiles>

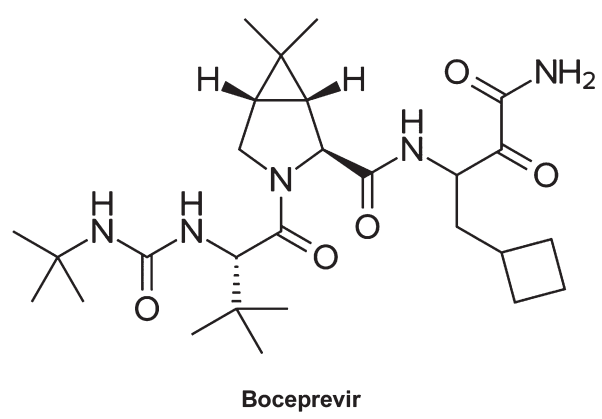<smiles>COc1ccc2c(O[C@@H]3C[C@H](C(=O)O)C(=O)N[C@]4(C(=O)O)C[C@@H]4/C=C\CCCCC[C@H](NC(=O)OC4CCCC4)C(=O)N4C[C@@H]4C3)cc(-c3csc(NC(C)C)n3)nc2c1</smiles>

BILN 2061

Fig. 1. Structures of Known HCV NS3-4A Serine Protease Inhibitors boxylic group.

\section{Results and Discussion}

Chemistry A convergent synthesis was designed for the preparation of series $\mathbf{4 a}-\mathbf{h}$ and $\mathbf{5 a}-\mathbf{h}$. The first part of this synthesis involved the preparation of 2-chloromethyl-1Hbenzo $[d]$ imidazole $\mathbf{1}$ as previously $\operatorname{reported}^{30,31)}$ (Chart 1). The second part of this synthesis involved the preparation of different amino and mercapto pyrimidines $\mathbf{2} \mathbf{a}-\mathbf{h}$ and $\mathbf{3} \mathbf{a}-\mathbf{h}$ by ternary condensation of the appropriate aldehyde with thiourea or guanidine hydrochloride and ethylcyanoacetate according to reported procedures ${ }^{30,32-34)}$ (Chart 1). Aiming to study the influence of substitution at different positions of the phenyl ring linked to pyrimidine, different substituted benzaldehydes were used to obtain the two series $\mathbf{2 a}-\mathbf{f}$ and $\mathbf{3 a}-\mathbf{f}$. In order to investigate the effect of the replacement of the phenyl ring by five membered heterocycles, 2-thiophenecarbaldehyde or 2-furancarbaldehyde were chosen to produce the desired pyrimidines $\mathbf{2 g}, \mathbf{h}$ and $\mathbf{3 g}, \mathbf{h}$ respectively. Dehydrohalogenation between the two parts was then carried out in basic medium to obtain the required target compounds $\mathbf{4 a}-\mathbf{h}$ and $\mathbf{5 a}-\mathbf{h}$, which were subsequently hydrolyzed by refluxing with $80 \%$ $\mathrm{H}_{2} \mathrm{SO}_{4}$ acid to obtain the carboxylic acid derivatives $\mathbf{6 a}-\mathbf{h}$ and 7a-h (Chart 2).

Inhibition of HCV Viral RNA Replication As a first experiment compounds $\mathbf{4 a}$ and $\mathbf{5 a}$ were tested for their $\mathrm{HCV}$ inhibitory activity (Table 1 , entries 2 and 10). Both $\mathrm{IC}_{50}$ and $\mathrm{IC}_{90}$ were evaluated. VX-950 was used as a standard. It was found<smiles>Cn1c(Cc2nc3ccc(C(N)=O)cc3[nH]2)nc2ccc(C(=O)NC(CP(=O)(O)O)C(=O)O)cc21</smiles>

Fig. 2. Structure of CRA-6336<smiles>[X]/C(N)=[14C]\C(=[OH+])C(=O)OCC</smiles><smiles></smiles> 

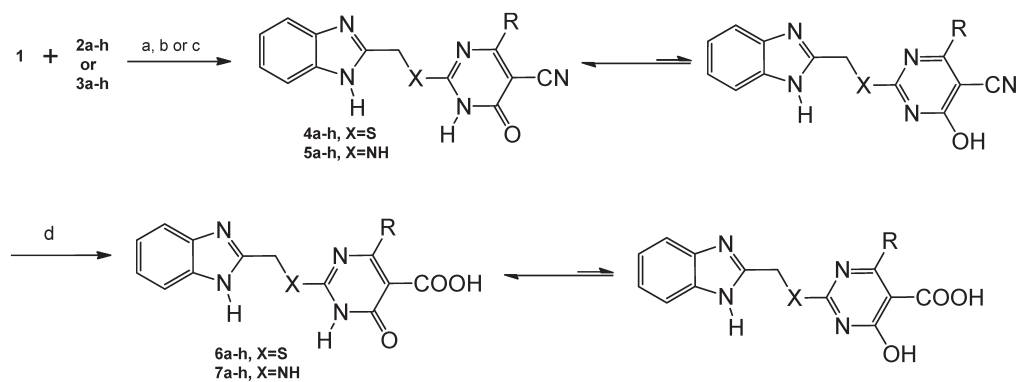

Reagents and conditions: a) triethylamine, tetrahydrofuran, $24 \mathrm{~h}, 73-81 \%$; b) $\mathrm{K}_{2} \mathrm{CO}_{3}$, acetone, rt, $10 \mathrm{~h}$ then reflux for $6 \mathrm{~h}, 73-81 \%$; $) \mathrm{K}_{2} \mathrm{CO}_{3}$, DMF, rt, $2 \mathrm{~h}$ then reflux for

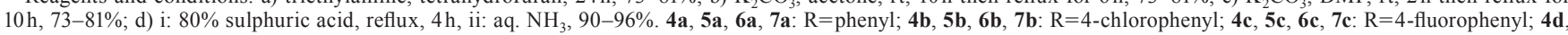
5d, 6d, 7d: R=4-hydroxyphenyl; 4e, 5e, 6e, 7e: R=2-chlorophenyl; 4f, 5f, 6f, 7f: R=2-hydroxyphenyl; 4g, 5g, 6g, 7g: R=2-thienyl; 4h, 5h, 6h, 7h: R=2-furyl.

Chart 2

Table 1. The Results of HCV RNA Replication Inhibitory Activity $\left(\mathrm{IC}_{50}, \mathrm{IC}_{90}\right)(\mu \mathrm{M})$ and the Cytotoxic Activity $\mathrm{CC}_{50}(\mu \mathrm{M})$ of the Tested Compounds and Standard

\begin{tabular}{|c|c|c|c|c|}
\hline Entry & Product & $\mathrm{IC}_{50}{ }^{a)}( \pm$ S.D. $) \mu \mathrm{M}$ after $48 \mathrm{~h}$ & $\mathrm{IC}_{90}{ }^{b)}( \pm$ S.D. $) \mu \mathrm{M}$ after $48 \mathrm{~h}$ & $\mathrm{CC}_{50}^{c)}( \pm$ S.D. $) \mu \mathrm{M}$ \\
\hline 1 & 1 & $0.25 \pm 0.02$ & $0.65 \pm 0.05$ & $137 \pm 6$ \\
\hline 2 & $4 a$ & $0.345 \pm 0.04$ & $0.744 \pm 0.06$ & $245 \pm 13$ \\
\hline 3 & $4 b$ & $0.312 \pm 0.03$ & $0.654 \pm 0.05$ & $453 \pm 18$ \\
\hline 4 & $4 c$ & $0.231 \pm 0.01$ & $0.563 \pm 0.04$ & $245 \pm 8$ \\
\hline 5 & 4d & $0.123 \pm 0.02$ & $0.321 \pm 0.05$ & $265 \pm 9$ \\
\hline 6 & $4 e$ & $0.245 \pm 0.05$ & $0.564 \pm 0.05$ & $312 \pm 11$ \\
\hline 7 & $4 f$ & $0.145 \pm 0.04$ & $0.345 \pm 0.04$ & $311 \pm 12$ \\
\hline 8 & $4 g$ & $0.355 \pm 0.03$ & $0.765 \pm 0.07$ & $456 \pm 15$ \\
\hline 9 & $4 h$ & $0.129 \pm 0.02$ & $0.432 \pm 0.07$ & $657 \pm 19$ \\
\hline 10 & $5 \mathbf{a}$ & $0.263 \pm 0.02$ & $0.678 \pm 0.05$ & $765 \pm 19$ \\
\hline 11 & $5 \mathbf{b}$ & $0.235 \pm 0.02$ & $0.456 \pm 0.05$ & $986 \pm 25$ \\
\hline 12 & $5 c$ & $0.277 \pm 0.02$ & $0.654 \pm 0.04$ & $643 \pm 22$ \\
\hline 13 & $6 a$ & $0.465 \pm 0.04$ & $1.12 \pm 0.11$ & $564 \pm 30$ \\
\hline 14 & $6 \mathbf{b}$ & $0.356 \pm 0.03$ & $0.879 \pm 0.09$ & $523 \pm 34$ \\
\hline 15 & $6 c$ & $0.277 \pm 0.02$ & $0.678 \pm 0.07$ & $545 \pm 33$ \\
\hline 16 & $6 d$ & $0.116 \pm 0.01$ & $0.452 \pm 0.05$ & $656 \pm 32$ \\
\hline 17 & $6 e$ & $0.298 \pm 0.02$ & $0.666 \pm 0.04$ & $978 \pm 47$ \\
\hline 18 & $6 f$ & $0.285 \pm 0.02$ & $0.987 \pm 0.09$ & $766 \pm 43$ \\
\hline 19 & $6 \mathrm{~g}$ & $0.255 \pm 0.02$ & $0.764 \pm 0.07$ & $587 \pm 12$ \\
\hline 20 & $6 \mathrm{~h}$ & $0.181 \pm 0.01$ & $0.535 \pm 0.06$ & $876 \pm 16$ \\
\hline 21 & $7 c$ & $0.546 \pm 0.05$ & $1.23 \pm 0.1$ & $647 \pm 23$ \\
\hline 22 & $7 d$ & $0.512 \pm 0.05$ & $0.987 \pm 0.1$ & $987 \pm 32$ \\
\hline 23 & $7 e$ & $0.546 \pm 0.05$ & $1.43 \pm 0.14$ & $953 \pm 33$ \\
\hline 24 & $7 f$ & $0.657 \pm 0.06$ & $1.78 \pm 0.16$ & $673 \pm 17$ \\
\hline 25 & $7 \mathrm{~h}$ & $0.87 \pm 0.08$ & $2.12 \pm 0.2$ & $964 \pm 24$ \\
\hline 26 & VX-950 $\left.{ }^{d}\right)$ & $0.20 \pm 0.0001$ & $0.45 \pm 0.0003$ & $90 \pm 1.2$ \\
\hline
\end{tabular}

a) Compound concentration required to achieve $50 \%$ inhibition of HCV RNA replication. $b$ ) Compound concentration required to achieve $90 \%$ inhibition of HCV RNA replication. c) Compound concentration required to reduce the cell viability by $50 \%$ as determined by MTT method. $d$ ) The standard used in comparison. $*$ Data expressed as means \pm S.D. for three independent experiments.

that both compounds $\mathbf{4 a}$ and $\mathbf{5 a}$ have excellent inhibitory activities $\left(\mathrm{IC}_{50 / 90}=0.345 / 0.744 \mu \mathrm{M}, \mathrm{IC}_{50 / 90}=0.263 / 0.678 \mu \mathrm{M}\right.$, respectively) when compared with VX-950 $\left(\mathrm{IC}_{50 / 90}=0.20 / 0.45 \mu \mathrm{M}\right)$ (Table 1, entry 26). The cytotoxic activity of both compounds was also tested against the viability hepatocyte. It was found that $4 \mathbf{a}$ and $5 \mathbf{a}\left(50 \%\right.$ cytotoxic concentration $\mathrm{CC}_{50}=245 \mu \mathrm{M}$ and $\mathrm{CC}_{50}=765 \mu \mathrm{M}$, respectively) (Table 1, entries 2 and 10) have lower cytotoxic activity when compared with VX-950 which has a value of $\mathrm{CC}_{50}=90 \mu \mathrm{M}$ (Table 1, entry 26). This result revealed the perfect selectivity of the synthesized scaffold against the virus in comparison to the host cell. After the successful finding of the HCV inhibitory activity of these two compounds $\mathbf{4 a}$ and $\mathbf{5 a}$, different modifications were carried out and some of the synthesized compounds were further tested for their anti-HCV activity and for their cytotoxic activity. It was found that all of the tested compounds have significant activity. Comparing the inhibitory activity values revealed that compounds $\mathbf{4 d}$, f, and $\mathbf{h}$ were more potent than the standard. It was also observed that bioisosteric replacement of $\mathrm{CH}_{2}-\mathrm{S}$ linkage in series $4 \mathbf{a}-\mathbf{h}$ and $\mathbf{6 a}-\mathbf{h}$ by $\mathrm{CH}_{2}-\mathrm{NH}$ bridge in series $\mathbf{5 a}-\mathbf{h}$ and $7 \mathbf{a}-\mathbf{h}$, resulted in a decrease or total loss of activity except in case of $\mathbf{5 b}$ where high potency was observed. The $\mathrm{IC}_{50 / 90}$ values of $\mathbf{4 c}, \mathbf{6 c}$ (Table 1 , entries 4 and 15) were $0.231 / 0.563$ and $0.277 / 0.678 \mu \mathrm{M}$, respectively while the $\mathrm{IC}_{50 / 90}$ values of 5c, 7c (Table 1, entries 12 and 21) were 0.277/0.654 and $0.546 / 1.23 \mu \mathrm{M}$, respectively. The effect of hydrolyzing the 

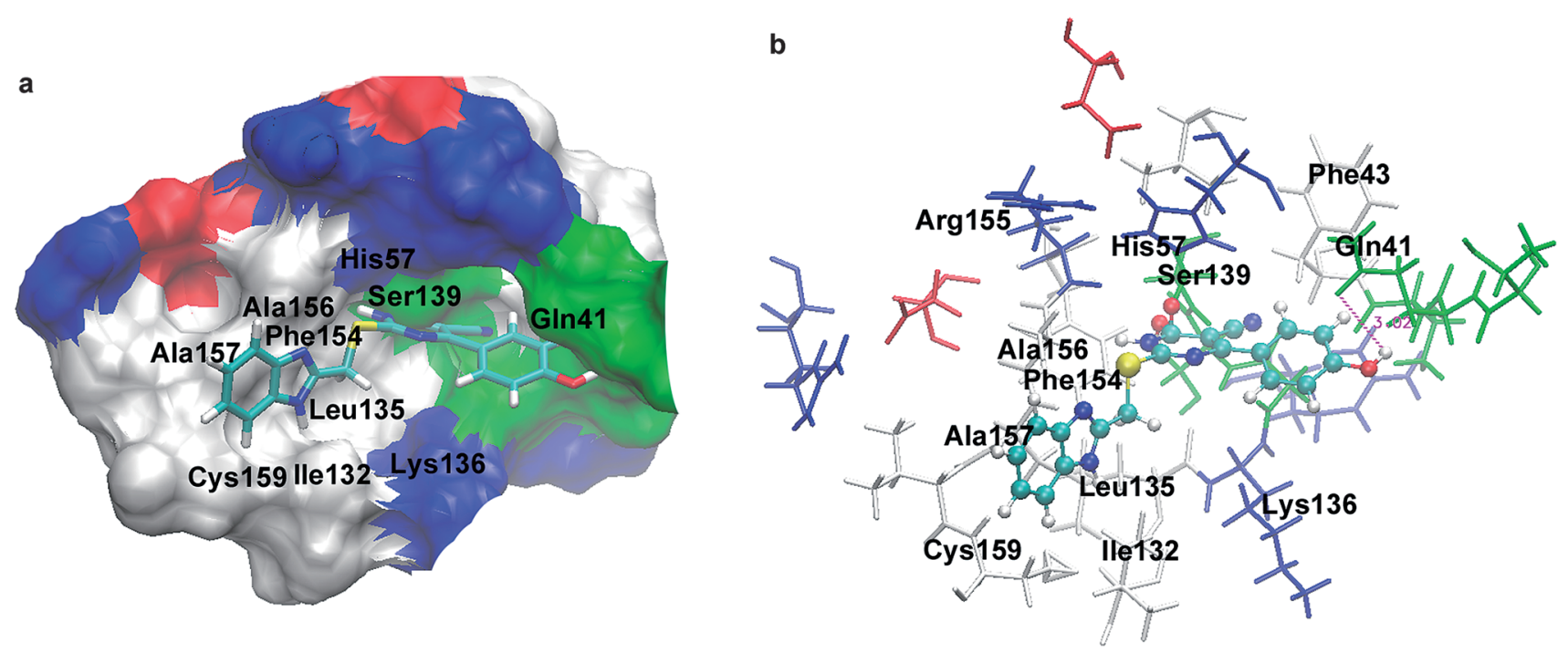

Fig. 3. Docking Pose of $\mathbf{4 d}$ in the Binding Site of NS3-4A Serine Protease (PDB Code 2FM2)

(a) Solid surface representation of NS3 active site with 4d (stick model, colored by atom type). On the protein surface, acidic residues are colored in red, basic residues are in blue, hydrophobic residues are in white, polar residues are in green. (b) The NS3 residues are shown as stick models and colored by residue type, the structure of $\mathbf{4 d}$ is presented as CPK model.

a

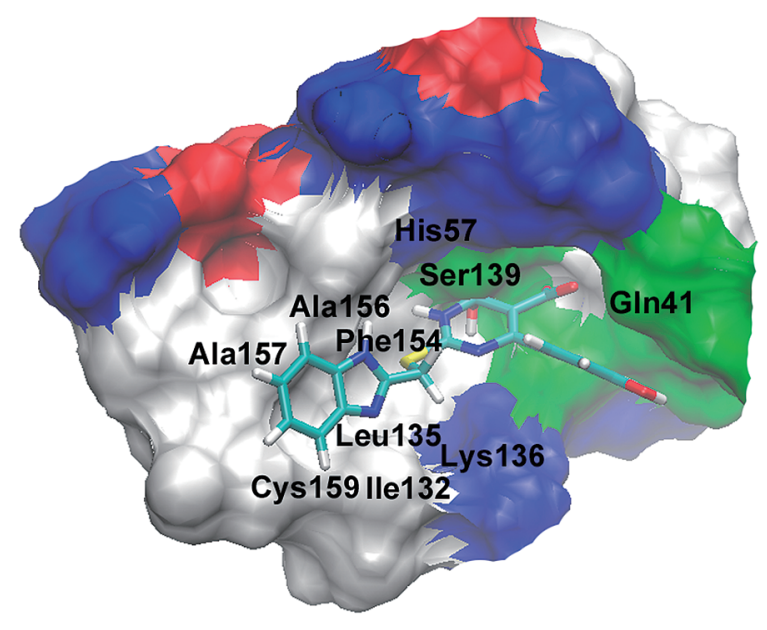

b

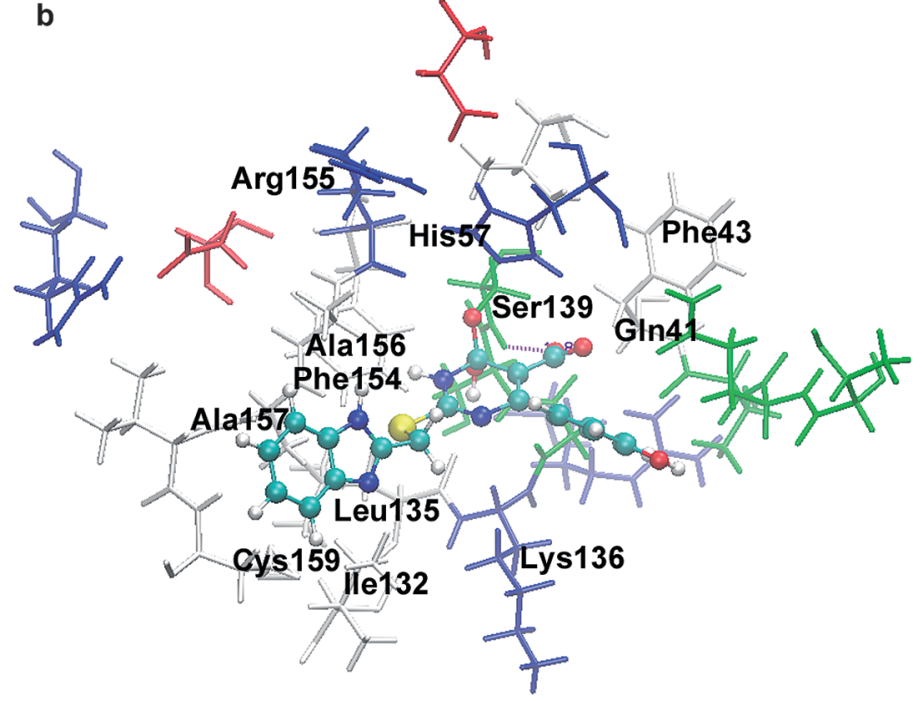

Fig. 4. Docking Pose of 6d in the Binding Site of NS3-4A Serine Protease (PDB Code 2FM2)

(a) Solid surface representation of NS3 active site with 6d (stick model, colored by atom type). On the protein surface, acidic residues are colored in red, basic residues are in blue, hydrophobic residues are in white, polar residues are in green. (b) The NS3 residues are shown as stick models and colored by residue type, the structure of $\mathbf{6 d}$ is presented as CPK model.

cyano group in series $\mathbf{4 a}-\mathbf{h}$ and $\mathbf{5 a}-\mathbf{h}$ to give series $\mathbf{6 a}-\mathbf{h}$ and $7 \mathbf{a}-\mathbf{h}$, respectively on the inhibitory activity was also studied. Comparing the $\mathrm{IC}_{50 / 90}$ of $\mathbf{4 a}-\mathbf{h}$ and $\mathbf{5 c}$ (Table 1, entries 2-9, 12) with the $\mathrm{IC}_{50 / 90}$ of $\mathbf{6} \mathbf{a}-\mathbf{h}$ and $7 \mathbf{c}$ (Table 1, entries 13-21) revealed the higher activity of the cyano containing series $(\mathbf{4 a}-\mathbf{h}$ and $\mathbf{5 c})$. This can be attributed to the steric demanding effect of the carboxylic group in $\mathbf{6 a}-\mathbf{h}$ and $7 \mathbf{c}$ which hinders the perfect fitting to the enzyme and also the cyano group is positioned in a hydrophobic area in the binding site. The only exceptions were $\mathbf{4 d}$ and $\mathbf{4 g}$ (Table 1 , entries 5 and 8) in which compounds 6d and $\mathbf{6 g}$ (Table 1, entries 16 and 19) having a carboxylic substituent showed higher activity. Subsequently, the effect of different substituents in the 6-position of the pyrimidine moiety was examined in each series. It was found that the compounds having $p$-hydroxyphenyl group namely, 4d, 6d, 7d exhibited higher activities when compared with the corresponding substitutions in the same series (Table 1, entries 5, 16, and 22). This may be due to hydrogen bonding of the $\mathrm{OH}$ group with the enzyme. The higher potency of the 4-hydroxy derivatives $\mathbf{4 d}, \mathbf{6 d}$, and $\mathbf{7 d}$ than the 2-hydroxy derivatives $\mathbf{4 f}$, $6 \mathbf{f}$, and $\mathbf{7 f}$, respectively, may be due to more favorable steric factors and best fitting with the enzyme. By comparing all of the tested compounds with VX-950 as a standard, we found that compounds $4 \mathbf{d}, \mathbf{4 f}, \mathbf{4 h}, \mathbf{6 d}, \mathbf{6 h}$ showed higher inhibitory activity than the standard (Table 1 , entries 5 , $7,9,16$, and 20).

We have also evaluated the $\mathrm{CC}_{50}$ of all the tested compounds against hepatocytes. It was found that all of the tested compounds have a lower cytotoxic activity when compared with VX-950 (Table 1). 
Molecular Modeling Calculations In order to rationalize the described biological results, docking studies of compounds 4d and 6d were undertaken. The program GOLD 5.0.1 $1^{35-37)}$ with the GoldScore fitness function, which uses bond strengths, was used for the docking calculations.

Analysis of key protein-ligand contacts and binding affinity of the poses provides insight into binding of $\mathbf{4 d}$ and $\mathbf{6 d}$ to the active sites of NS3-4A serine protease.

For the lowest energy poses of $\mathbf{4 d}$, hydrogen bond interactions were observed between the $p-\mathrm{OH}$ and $\mathrm{Gln} 41$ (3.08 $\AA$ ). This was consistent with the observed SAR (Figs. 3a, b).

For the lowest energy poses of $\mathbf{6 d}$, both oxygens of the carboxylate forms $\mathrm{H}$ bond with the $\mathrm{NH}$ group of backbone of Ser139 (ca. 1.8, 2.3 $\AA$ ) and with the $\mathrm{NH}$ group of the backbone of Gly137 ( $c a .3 \AA$ ). The $p$-OH has a distance of $3.38 \AA$ from Gln41 (Figs. 4a, b). The binding to Ala156 residue is vital for all the NS3-4A inhibitors. ${ }^{38)}$

The $\mathrm{IC}_{50 / 90}$ of $\mathbf{4 d}(0.123 / 0.321 \mu \mathrm{M})$ compared to that of VX-950 $(0.20 / 0.45 \mu \mathrm{M})$ may be attributed to the novel feature of $\mathbf{4 d}$, it extends substantially toward the $\mathbf{P}^{\prime}$ side of the active site. Compound $\mathbf{4 d}$ derives a significant part of its binding energy from contacts on both $\mathbf{P}$ and $\mathbf{P}^{\prime}$ sides. Interaction with Lys136 of the protein may be a reason for the improved potency of this inhibitor.

These additional interactions of compound $\mathbf{4 d}$ versus VX-950 justify its higher activity. The active site pockets that compound 4d occupy are depicted in Figs. 3a and b.

The residues that induce common interactions with both $\mathbf{4 d}$ and 6d are Lys136, Ser139, Phe154, Ala156, Ala157, Arg155, and Cys159 (Figs. 3, 4). Those residues are common in NS3-4A inhibitor binding as it has been widely discussed in previous literature. $^{21,39)}$ As previously reported, hydrophobic interactions highly contribute in the binding affinity of the majority of NS3-4A serine protease inhibitors. The residues Ile132, Leu135, Lys136, Phe154, Ala156, Ala157, and Cys159 play important roles in hydrophobic binding.

The higher activity of cyanopyrimidines compared to their carboxy analogues may be attributed to that they are directed towards a hydrophobic area in the binding site (Phe43).

As shown in Figs. 3a and 4a, it was observed that methylenethio bridge fitted into the hydrophobic cavity formed by Ala156, Ile132, Leu135, and Phe154. This may explain why compounds which have methylenethio bridge have higher inhibitory activity than compounds which have methyleneamino bridge.

\section{Conclusion}

Successfully, through the present study, a new series of potent HCV inhibitors having the benzimidazole-2-yl-methylthiopyrimidine scaffold with high potency was discovered. The docking results showed that the 2-(4-hydroxyphenyl)pyrimidine is a good scaffold to be explored, and that 4-positioned substituents that can perform $\mathrm{H}$-bonding interactions with the enzyme are important.

The most active compound $\mathbf{4 d}$ gave better results than Telaprevir (VX-950). The presence of the 4-OH on the phenyl ring linked to pyrimidine in compound $\mathbf{4 d}$ was found to have great impact on activity. The new potent compounds need further pharmacological investigation to be used as HCV inhibitors in the near future.

\section{Experimental}

Chemistry Melting points are uncorrected and were recorded on a Gallenkamp thermometer melting point apparatus. IR spectra $(\mathrm{KBr})$ were recorded on Bruker Vector 22 and JASCO FT/IR 300E Fourier transformer spectrophotometer. NMR spectra were recorded on Varian GEMINI 200 $(200 \mathrm{MHz})$ and JEOL EX-270 and $500 \mathrm{MHz}$ spectrometers using DMSO- $d_{6}$ as a solvent. Mass spectra were recorded on FINNGAN MAT SSQ 7000. Elemental analysis was performed in the microanalytical laboratory of the National Research Center. The synthesized compounds were named using ChemDraw Ultra software (v 10.0).

Preparation of 2-((1H-Benzo[d]imidazol-2-yl)methylthio)1,6-dihdyro-4-(aryl or heteroaryl)-6-oxopyrimidine-5-carbonitriles $(4 a-f))$. General Method A solution of the appropriate thiouracil $\mathbf{2 a}-\mathbf{f}^{32,33)}(42.17 \mathrm{mmol})$ in dry tetrahydrofuran $(60 \mathrm{~mL})$ containing triethylamine $(0.5 \mathrm{~mL})$ was stirred for $1 \mathrm{~h}$. A solution of $1(7 \mathrm{~g}, 42.17 \mathrm{mmol})$ in dry tetrahydrofuran was then added portionwise and the reaction mixture was allowed to stir at room temperature for additional $24 \mathrm{~h}$, poured onto crushed ice with stirring. The precipitated product was filtered off and recrystallized from the appropriate solvent.

2-((1H-Benzo[d]imidazol-2-yl)methylthio)-1,6-dihydro-6oxo-4-phenylpyrimidine-5-carbonitrile (4a) was prepared according to the reported method. ${ }^{30)}$

2-((1H-Benzo[d]imidazol-2-yl)methylthio)-4-(4-chlorophenyl)-1,6-dihydro-6-oxopyrimidine-5-carbonitrile (4b): Yield $75 \%$; mp $183-185^{\circ} \mathrm{C}$ (from $\mathrm{MeOH}$ ); ${ }^{1} \mathrm{H}-\mathrm{NMR}(500 \mathrm{MHz}$, DMSO- $\left.d_{6}\right) \delta: 4.67\left(\mathrm{~s}, 2 \mathrm{H}, \mathrm{CH}_{2}-\mathrm{S}\right), 7.18-7.22(\mathrm{~m}, 2 \mathrm{H}, \mathrm{H} 5$ and $\mathrm{H} 6$ benzimidazole), 7.28-7.33 (m, 2H, H3' and $\mathrm{H}^{\prime}$ phenyl), 7.50-7.55 (m, 2H, H4 and $\mathrm{H} 7$ benzimidazole), 7.87-7.91 (m, $2 \mathrm{H}, \mathrm{H} 2^{\prime}$ and $\mathrm{H6}^{\prime}$ phenyl), 8.21 (br, $1 \mathrm{H}, \mathrm{NH}$ pyrimidine, $\mathrm{D}_{2} \mathrm{O}$ exchangeable), 10.17 (br, $1 \mathrm{H}, \mathrm{NH}$ benzimidazole, $\mathrm{D}_{2} \mathrm{O}$ exchangeable); IR ( $\mathrm{KBr}, \mathrm{cm}^{-1}$ ) 3394 ( $\mathrm{NH}$ benzimidazole), 3125 ( $\mathrm{NH}$ pyrimidine), 3056 ( $\mathrm{CH}$ arom.), 2924 (CH aliph.), 2209 $(\mathrm{CN}), 1647(\mathrm{C}=\mathrm{O}), 1629(\mathrm{C}=\mathrm{N}$ benzimidazole), 1552, 1518 $(\mathrm{C}=\mathrm{N}$ pyrimidine, $\mathrm{C}=\mathrm{C}$ ). $\mathrm{MS}$ (electron ionization $(\mathrm{EI})$ ) $\mathrm{m} / \mathrm{z}$ 393/395 ( $\mathrm{M}^{+}$, 19\%/6\% Cl pattern), 180 (100\%). Anal. Calcd for $\mathrm{C}_{19} \mathrm{H}_{12} \mathrm{ClN}_{5} \mathrm{OS}$ : C, 57.94; H, 3.08; N, 17.78; S, 8.14. Found: C, 57.75; H, 3.00; N, 17.57; S, 8.36.

2-((1H-Benzo[d]imidazol-2-yl)methylthio)-4-(4-fluorophenyl)-1,6-dihydro-6-oxopyrimidine-5-carbonitrile (4c): Yield $76 \%$; mp $250-252^{\circ} \mathrm{C}$ (from $\mathrm{MeOH}$ ); ${ }^{1} \mathrm{H}-\mathrm{NMR} \quad(500 \mathrm{MHz}$, DMSO- $\left.d_{6}\right) \delta: 4.79\left(\mathrm{~s}, 2 \mathrm{H}, \mathrm{CH}_{2}-\mathrm{S}\right), 7.22-7.26(\mathrm{~m}, 2 \mathrm{H}, \mathrm{H} 5$ and H6 benzimidazole), 7.30-7.39 ( $\mathrm{m}, 2 \mathrm{H}, \mathrm{H} 3^{\prime}$ and $\mathrm{H} 5^{\prime}$ phenyl), 7.55-7.60 ( $\mathrm{m}, 2 \mathrm{H}, \mathrm{H} 4$ and $\mathrm{H} 7$ benzimidazole), 7.91-7.98 (m, $2 \mathrm{H}, \mathrm{H} 2^{\prime}$ and $\mathrm{H6}^{\prime}$ phenyl), 10.04 (br, 2H, OH enolic and $\mathrm{NH}$ benzimidazole, $\mathrm{D}_{2} \mathrm{O}$ exchangeable); IR $\left(\mathrm{KBr}, \mathrm{cm}^{-1}\right) 3365$ (NH benzimidazole), 3200-2500 (br, enolic OH), $3051(\mathrm{CH}$ arom.), 2963 ( $\mathrm{CH}$ aliph.), $2212(\mathrm{CN}), 1628$ (C=N benzimidazole), 1530, $1505(\mathrm{C}=\mathrm{N}$ pyrimidine, $\mathrm{C}=\mathrm{C})$; $\mathrm{MS}(\mathrm{EI}) \mathrm{m} / \mathrm{z} 377$ $\left(\mathrm{M}^{+}, 3 \%\right), 379\left(\mathrm{M}^{+\cdot}+2,0.71 \%\right), 57(100 \%)$. Anal. Calcd for $\mathrm{C}_{19} \mathrm{H}_{12} \mathrm{FN}_{5} \mathrm{OS}$ : C, 60.47; H, 3.20; N, 18.56; S, 8.50. Found: C, 60.25; H, 3.45; N, 18.32; S, 8.24.

2-((1H-Benzo[d]imidazol-2-yl)methylthio)-1,6-dihydro-4-(4hydroxyphenyl)-6-oxopyrimidine-5-carbonitrile (4d): Yield $74 \%$; mp $200-202^{\circ} \mathrm{C}$ (from $\mathrm{MeOH}$ ); ${ }^{1} \mathrm{H}-\mathrm{NMR} \quad(500 \mathrm{MHz}$, DMSO- $\left.d_{6}\right) \delta: 4.50\left(\mathrm{~s}, 2 \mathrm{H}, \mathrm{CH}_{2}-\mathrm{S}\right), 7.01-7.20(\mathrm{~m}, 2 \mathrm{H}, \mathrm{H} 5$ and H6 benzimidazole), 7.31-7.67 (m, 4H, 4 phenyl H), 7.70-7.89 (m, 2H, H4 and $\mathrm{H} 7$ benzimidazole), 8.20 (br, 1H, NH py- 
rimidine, $\mathrm{D}_{2} \mathrm{O}$ exchangeable), $11.45(\mathrm{~s}, 1 \mathrm{H}, \mathrm{OH}$ phenolic, $\mathrm{D}_{2} \mathrm{O}$ exchangeable), $12.40\left(\mathrm{br}, 1 \mathrm{H}, \mathrm{NH}\right.$ benzimidazole, $\mathrm{D}_{2} \mathrm{O}$ exchangeable); IR ( $\mathrm{KBr}, \mathrm{cm}^{-1}$ ) 3351 ( $\mathrm{NH}$ benzimidazole), 3259 (br, NH pyrimidine, $\mathrm{H}$ bonded $\mathrm{OH}$ phenolic), $3063(\mathrm{CH}$ arom.), 2925 (CH aliph.), $2217(\mathrm{CN}), 1654(\mathrm{C}=\mathrm{O}), 1608(\mathrm{C}=\mathrm{N}$ benzimidazole), 1565, $1515(\mathrm{C}=\mathrm{N}, \mathrm{C}=\mathrm{C}$ pyrimidine); $\mathrm{MS}(\mathrm{EI})$ m/z $375\left(\mathrm{M}^{+*}, 2 \%\right), 377\left(\mathrm{M}^{+\cdot}+2,1 \%\right), 217$ (100\%). Anal. Calcd for $\mathrm{C}_{19} \mathrm{H}_{13} \mathrm{~N}_{5} \mathrm{O}_{2} \mathrm{~S}$ : C, 60.78; H, 3.50; N, 18.66; S, 8.54. Found: C, 60.54; H, 3.42; N, 18.34; S, 8.28.

2-((1H-Benzo[d]imidazol-2-yl)methylthio)-4-(2-chlorophenyl)-1,6-dihydro-6-oxopyrimidine-5-carbonitrile (4e): Yield $76 \%$; mp $210-212{ }^{\circ} \mathrm{C}$ (from $\mathrm{MeOH}$ ); ${ }^{1} \mathrm{H}-\mathrm{NMR}(500 \mathrm{MHz}$, DMSO- $\left.d_{6}\right) \delta: 4.51\left(\mathrm{~s}, 2 \mathrm{H}, \mathrm{CH}_{2}-\mathrm{S}\right), 7.45-7.58(\mathrm{~m}, 6 \mathrm{H}, \mathrm{H} 5$ and $\mathrm{H} 6$ benzimidazole +4 phenyl $\mathrm{H}), 7.70-7.88(\mathrm{~m}, 2 \mathrm{H}, \mathrm{H} 4$ and $\mathrm{H} 7$ benzimidazole), 8.01 (br, $1 \mathrm{H}, \mathrm{NH}$ pyrimidine, $\mathrm{D}_{2} \mathrm{O}$ exchangeable), 11.35 (br, 1H, $\mathrm{NH}$ benzimidazole, $\mathrm{D}_{2} \mathrm{O}$ exchangeable); IR $\left(\mathrm{KBr}, \mathrm{cm}^{-1}\right) 3387$ ( $\mathrm{NH}$ benzimidazole), 3136 (br, $\mathrm{NH}$ pyrimidine), 3022 (CH arom.), 2922 ( $\mathrm{CH}$ aliph.), $2204(\mathrm{CN})$, $1652(\mathrm{C}=\mathrm{O}), 1609(\mathrm{C}=\mathrm{N}$ benzimidazole $), 1561,1514(\mathrm{C}=\mathrm{N}$ pyrimidine, $\mathrm{C}=\mathrm{C}$ ); $\mathrm{MS}(\mathrm{EI}) \mathrm{m} / \mathrm{z} 393 / 395\left(\mathrm{M}^{+\cdot}, 10 \% / 4 \% \mathrm{Cl}\right.$ pattern); 164 (100\%). Anal. Calcd for $\mathrm{C}_{19} \mathrm{H}_{12} \mathrm{ClN}_{5} \mathrm{OS}$ : C, 57.94; H, 3.08; N, 17.78; S, 8.14. Found: C, 57.90; H, 2.95; N, 17.67; S, 7.78 .

2-((1H-Benzo[d]imidazol-2-yl)methylthio)-1,6-dihydro-4-(2hydroxyphenyl)-6-oxopyrimidine-5-carbonitrile (4f): Yield $73 \%$; mp $193-195^{\circ} \mathrm{C}$ (from $\mathrm{MeOH}$ ); ${ }^{1} \mathrm{H}-\mathrm{NMR} \quad(500 \mathrm{MHz}$, DMSO- $\left.d_{6}\right) \delta: 4.44\left(\mathrm{~s}, 2 \mathrm{H}, \mathrm{CH}_{2}-\mathrm{S}\right), 7.12-7.24(\mathrm{~m}, 2 \mathrm{H}, \mathrm{H} 5$ and H6 benzimidazole), 7.35-7.67 (m, 4H, 4 phenyl $\mathrm{H}$ ), 7.75-7.90 (m, 2H, H4 and $\mathrm{H} 7$ benzimidazole), 8.25 (br, 1H, NH pyrimidine, $\mathrm{D}_{2} \mathrm{O}$ exchangeable), $11.22\left(\mathrm{~s}, 1 \mathrm{H}, \mathrm{OH}\right.$ phenolic, $\mathrm{D}_{2} \mathrm{O}$ exchangeable), 12.52 (br, $1 \mathrm{H}, \mathrm{NH}$ benzimidazole, $\mathrm{D}_{2} \mathrm{O}$ exchangeable); IR ( $\mathrm{KBr}, \mathrm{cm}^{-1}$ ) 3354 ( $\mathrm{NH}$ benzimidazole), 3206 ( $\mathrm{NH}$ pyrimidine, $\mathrm{H}$ bonded $\mathrm{OH}$ phenolic), 3030 ( $\mathrm{CH}$ arom.), 2924 ( $\mathrm{CH}$ aliph.), $2216(\mathrm{CN}), 1664(\mathrm{C}=\mathrm{O}), 1627(\mathrm{C}=\mathrm{N}$ benzimidazole), 1588, $1558(\mathrm{C}=\mathrm{N}$ pyrimidine, $\mathrm{C}=\mathrm{C})$. Anal. Calcd for $\mathrm{C}_{19} \mathrm{H}_{13} \mathrm{~N}_{5} \mathrm{O}_{2} \mathrm{~S}$ : C, 60.79; H, 3.49; N, 18.66; S, 8.54. Found: C, 60.57; H, 3.35; N, 18.42; S, 8.34

General Method for Preparation of $4 \mathbf{g}, \mathbf{h}$ To a well stirred mixture of the appropriate thiouracil $\mathbf{2 g}, \mathbf{h}^{32,33)}$ $(12.05 \mathrm{mmol})$ and anhydrous potassium carbonate $(1.66 \mathrm{~g}$, $12.05 \mathrm{mmol})$ in dry acetone $(25 \mathrm{~mL})$, a solution of $\mathbf{1}(2 \mathrm{~g}$, $12.05 \mathrm{mmol}$ ) in dry acetone was added portion wise. The reaction mixture was stirred for $10 \mathrm{~h}$ at room temperature then heated under reflux for additional $6 \mathrm{~h}$, cooled, filtered and the solid was washed with acetone. The organic solvent was evaporated under reduced pressure and the residue was recrystallized from the appropriate solvent.

2-((1H-Benzo[d]imidazol-2-yl)methylthio)-1,6-dihydro-6oxo-4-(thiophen-2-yl)pyrimidine-5-carbonitrile (4g): Yield 81\%; mp $200-202^{\circ} \mathrm{C}$ (from EtOH); ${ }^{1} \mathrm{H}-\mathrm{NMR}$ (200 MHz, DMSO$\left.d_{6}\right) \delta: 4.80\left(\mathrm{~s}, 2 \mathrm{H}, \mathrm{CH}_{2}-\mathrm{S}\right), 7.16-7.24\left(\mathrm{~m}, 2 \mathrm{H}, \mathrm{H}^{\prime}\right.$ and $\mathrm{H}^{\prime}$ thienyl), 7.28-7.33 (m, 1H, H5' thienyl), 7.51-7.58 (m, 2H, H5 and $\mathrm{H} 6$ benzimidazole), $7.98-8.12$ ( $\mathrm{m}, 2 \mathrm{H}, \mathrm{H} 4$ and $\mathrm{H} 7$ benzimidazole), 8.50 (br, $1 \mathrm{H}, \mathrm{NH}$ pyrimidine, $\mathrm{D}_{2} \mathrm{O}$ exchangeable), 12.20 (br, $1 \mathrm{H}, \mathrm{NH}$ benzimidazole, $\mathrm{D}_{2} \mathrm{O}$ exchangeable); IR $\left(\mathrm{KBr}, \mathrm{cm}^{-1}\right) 3380$ (NH benzimidazole), 3156 (NH pyrimidine), 3059 (CH arom.), 2970 (CH aliph.), 2209 (CN), $1680(\mathrm{C}=\mathrm{O})$, $1621(\mathrm{C}=\mathrm{N}$ benzimidazole $), 1550,1525(\mathrm{C}=\mathrm{N}$ pyrimidine, $\mathrm{C}=$ C); MS (EI) $m / z 365\left(\mathrm{M}^{+}, 2 \%\right), m / z 84$ (100\%). Anal. Calcd for $\mathrm{C}_{17} \mathrm{H}_{11} \mathrm{~N}_{5} \mathrm{OS}_{2}$ : C, 55.87; H, 3.04; N, 19.17; S, 17.55. Found: C, 55.47; H, 3.43; N, 19.28; S, 17.60 .
2-((1H-Benzo[d]imidazol-2-yl)methylthio)-4-(furan-2yl)-1,6-dihydro-6-oxopyrimidine-5-carbonitrile (4h): Yield $80 \%$; mp $223-225^{\circ} \mathrm{C}$ (from EtOH); ${ }^{1} \mathrm{H}-\mathrm{NMR} \quad(200 \mathrm{MHz}$, DMSO- $\left.d_{6}\right) \delta: 7.12-7.20\left(\mathrm{~m}, 2 \mathrm{H}, \mathrm{H} 3^{\prime}\right.$ and $\mathrm{H} 4^{\prime}$ furyl), 7.25-7.29 (m, 1H, H5' furyl), 7.48-7.52 (m, 2H, H5 and H6 benzimidazole), 7.88-8.02 (m, 2H, H4 and $\mathrm{H} 7$ benzimidazole), 8.42 (br, $1 \mathrm{H}, \mathrm{NH}$ pyrimidine, $\mathrm{D}_{2} \mathrm{O}$ exchangeable), $12.14(\mathrm{br}, 1 \mathrm{H}, \mathrm{NH}$ benzimidazole, $\mathrm{D}_{2} \mathrm{O}$ exchangeable); IR $\left(\mathrm{KBr}, \mathrm{cm}^{-1}\right) 3446(\mathrm{NH}$ benzimidazole), 3139 ( $\mathrm{NH}$ pyrimidine), 3034 (CH arom.), 2913 (CH aliph.), $2216(\mathrm{CN}), 1655(\mathrm{C}=\mathrm{O}), 1620(\mathrm{C}=\mathrm{N}$ benzimidazole), 1599, $1521(\mathrm{C}=\mathrm{N}$ pyrimidine, $\mathrm{C}=\mathrm{C}) ; \mathrm{MS}(\mathrm{EI}) \mathrm{m} / z 349$ $\left(\mathrm{M}^{+\cdot}, 1 \%\right), m / z 351\left(\mathrm{M}^{+\cdot}+2,1 \%\right), m / z 55$ (100\%). Anal. Calcd for $\mathrm{C}_{17} \mathrm{H}_{11} \mathrm{~N}_{5} \mathrm{O}_{2} \mathrm{~S}$ : C, 58.44; H, 3.18; N, 20.05; S, 9.18. Found: C, 58.30; H, 3.47; N, 20.25; S, 9.23.

Preparation of 2-((1H-Benzo[d]imidazol-2-yl)methylamino)-1,6-dihydro-4-(Aryl or Heteroaryl)-6-oxopyrimidine-5-carbonitriles (5a-h). General Method A solution of compound $\mathbf{1}(2 \mathrm{~g}, 12.05 \mathrm{mmol})$ in dry dimethylformamide $(7 \mathrm{~mL})$, was added to a mixture of the appropriate aminopyrimidine $\mathbf{3 a}-\mathbf{h}(12.05 \mathrm{mmol})$ and anhydrous potassium carbonate $(1.66 \mathrm{~g}, 12.05 \mathrm{mmol})$ in dimethylformamide $(10 \mathrm{~mL})$. The reaction mixture was allowed to stir for $2 \mathrm{~h}$ at room temperature then heated under reflux for additional $10 \mathrm{~h}$. After cooling, it was poured into ice-water with continuous stirring; the formed precipitate was filtered and recrystallized from the appropriate solvent.

2-((1H-Benzo $[d]$ imidazol-2-yl)methylamino)-1,6-dihydro-6oxo-4-phenyl pyrimidine-5-carbonitrile (5a): Yield 78\%; mp $116-118^{\circ} \mathrm{C}$ (from EtOH); ${ }^{1} \mathrm{H}-\mathrm{NMR}\left(200 \mathrm{MHz}, \mathrm{DMSO}-d_{6}\right) \delta$ : 5.25 (s, 2H, $\mathrm{CH}_{2}-\mathrm{NH}$ ), 6.47 (br, $1 \mathrm{H}, \mathrm{CH}_{2}-\mathrm{NH}, \mathrm{D}_{2} \mathrm{O}$ exchangeable), 7.38-8.49 (m, 9H, Ar-H), 9.81 (br, $1 \mathrm{H}, \mathrm{NH}$ pyrimidine, $\mathrm{D}_{2} \mathrm{O}$ exchangeable), $11.74\left(\mathrm{br}, 1 \mathrm{H}, \mathrm{NH}\right.$ benzimidazole, $\mathrm{D}_{2} \mathrm{O}$ exchangeable); IR ( $\mathrm{KBr}, \mathrm{cm}^{-1}$ ) 3388 ( $\mathrm{NH}$ benzimidazole), $3306\left(\underline{\mathrm{NH}}-\mathrm{CH}_{2}\right), 3137$ ( $\mathrm{NH}$ pyrimidine), 3051 ( $\mathrm{CH}$ arom.), 2928 ( $\overline{\mathrm{CH}}$ aliph.), $2210(\mathrm{CN}), 1688(\mathrm{C}=\mathrm{O}), 1625(\mathrm{C}=\mathrm{N}$ benzimidazole), 1593, $1517(\mathrm{C}=\mathrm{N}$ pyrimidine, $\mathrm{C}=\mathrm{C})$; $\mathrm{MS}(\mathrm{EI}) \mathrm{m} / \mathrm{z}$ $342\left(\mathrm{M}^{+}, 1 \%\right), m / z 91$ (100\%). Anal. Calcd for $\mathrm{C}_{19} \mathrm{H}_{14} \mathrm{~N}_{6} \mathrm{O}: \mathrm{C}$, 66.69; H, 4.09; N, 24.55. Found: C, 66.85; H, 4.34; N, 24.88.

2-((1H-Benzo[d]imidazol-2-yl)methylamino)-4-(4-chlorophenyl)-1,6-dihydro-6-oxopyrimidine-5-carbonitrile $\quad(\mathbf{5 b})$ : Yield 75\%; mp $195-197^{\circ} \mathrm{C}$ (from EtOH); ${ }^{1} \mathrm{H}-\mathrm{NMR}(200 \mathrm{MHz}$, DMSO- $\left.d_{6}\right) \delta: 5.76\left(\mathrm{~s}, 2 \mathrm{H}, \underline{\mathrm{CH}}_{2}-\mathrm{NH}\right), 5.88\left(\mathrm{br}, 1 \mathrm{H}, \mathrm{CH}_{2}-\underline{\mathrm{NH}}\right.$, $\mathrm{D}_{2} \mathrm{O}$ exchangeable), $6.60 \overline{(\mathrm{br},} 1 \mathrm{H}, \mathrm{NH}$ pyrimidine, $\mathrm{D}_{2} \mathrm{O} \overline{\mathrm{ex}}-$ changeable), 7.24 (d, $J=7.4 \mathrm{~Hz}, 2 \mathrm{H}, \mathrm{H} 3^{\prime}$ and $\mathrm{H}^{\prime}$ ' 4-Cl-phenyl), 7.35-7.43 (m, 2H, H5 and H6 benzimidazole), 7.63-7.75 (m, 2H, H4 and $\mathrm{H} 7$ benzimidazole), 7.89 (d, J=7.4 Hz, 2H, H2' and $\mathrm{H} 6^{\prime}$ 4-Cl-phenyl), 11.80 (br, $1 \mathrm{H}, \mathrm{NH}$ benzimidazole, $\mathrm{D}_{2} \mathrm{O}$ exchangeable); IR ( $\mathrm{KBr}, \mathrm{cm}^{-1}$ ) 3375 ( $\mathrm{NH}$ benzimidazole, NH- $\left.\mathrm{CH}_{2}\right), 3102$ (NH pyrimidine), 3051 ( $\mathrm{CH}$ arom.), $2980(\mathrm{CH}$ aliph.), 2212 (CN), $1671(\mathrm{C}=\mathrm{O}), 1619(\mathrm{C}=\mathrm{N}$ benzimidazole), 1597, $1517(\mathrm{C}=\mathrm{N}$ pyrimidine, $\mathrm{C}=\mathrm{C})$; $\mathrm{MS}(\mathrm{EI}) \mathrm{m} / \mathrm{z} 375\left(\mathrm{M}^{+}\right.$, 2\%), 164 (100\%). Anal. Calcd for $\mathrm{C}_{19} \mathrm{H}_{13} \mathrm{ClN}_{6} \mathrm{O}: \mathrm{C}, 60.56 ; \mathrm{H}$, 3.48; N, 22.31. Found: C, 60.86; H, 3.68; N, 22.64.

2-((1H-Benzo[d]imidazol-2-yl)methylamino)-4-(4-fluorophenyl)-1,6-dihydro-6-oxopyrimidine-5-carbonitrile $\quad(\mathbf{5 c})$ : Yield $80 \%$; mp $170-172{ }^{\circ} \mathrm{C}$ (from EtOH); ${ }^{1} \mathrm{H}-\mathrm{NMR}(200 \mathrm{MHz}$, DMSO-d $)_{6} \delta: 5.74\left(\mathrm{~s}, 2 \mathrm{H}, \underline{\mathrm{CH}}_{2}-\mathrm{NH}\right), 6.15\left(\mathrm{br}, 1 \mathrm{H}, \mathrm{CH}_{2}-\mathrm{NH}\right.$, $\mathrm{D}_{2} \mathrm{O}$ exchangeable), $7.22-7.30(\mathrm{~m}, 2 \mathrm{H}, \mathrm{H} 5$ and $\mathrm{H} 6$ benzimidazole), 7.60-7.72 (m, 4H, phenyl H), 7.93-8.10 (m, 2H, H4 and $\mathrm{H} 7$ benzimidazole), $8.98\left(\mathrm{br}, 1 \mathrm{H}, \mathrm{NH}\right.$ pyrimidine, $\mathrm{D}_{2} \mathrm{O}$ exchangeable), 12.82 (br, $1 \mathrm{H}, \mathrm{NH}$ benzimidazole, $\mathrm{D}_{2} \mathrm{O}$ ex- 
changeable); IR ( $\mathrm{KBr}, \mathrm{cm}^{-1}$ ) 3468 ( $\mathrm{NH}$ benzimidazole), 3323 $\left(\mathrm{NH}-\mathrm{CH}_{2}\right), 3201$ ( $\mathrm{NH}$ pyrimidine), 3056 ( $\mathrm{CH}$ arom.), 2926 ( $\overline{\mathrm{CH}}$ aliph.), $2201(\mathrm{CN}), 1678(\mathrm{C}=\mathrm{O}), 1624(\mathrm{C}=\mathrm{N}$ benzimidazole), 1598, $1555(\mathrm{C}=\mathrm{N}$ pyrimidine, $\mathrm{C}=\mathrm{C})$; $\mathrm{MS}(\mathrm{EI}) \mathrm{m} / \mathrm{z} 361$ $\left(\mathrm{M}^{+\cdot}+1,22 \%\right), m / z 260$ (100\%). Anal. Calcd for $\mathrm{C}_{19} \mathrm{H}_{13} \mathrm{FN}_{6} \mathrm{O}$ : C, 63.32; H, 3.64; N, 23.33. Found: C, 63.14; H, 3.30; N, 23.48.

2-((1H-Benzo[d]imidazol-2-yl)methylamino)-1,6-dihydro-4(4-hydroxyphenyl)-6-oxopyrimidine-5-carbonitrile (5d): Yield $78 \%$; mp $230-232^{\circ} \mathrm{C}$ (from $\mathrm{MeOH}$ ); ${ }^{1} \mathrm{H}-\mathrm{NMR} \quad(200 \mathrm{MHz}$, DMSO- $\left.d_{6}\right) \delta: 4.88$ (br, $1 \mathrm{H}, \mathrm{CH}_{2}-\underline{\mathrm{NH}}, \mathrm{D}_{2} \mathrm{O}$ exchangeable), $5.50\left(\mathrm{~s}, 2 \mathrm{H}, \mathrm{CH}_{2}-\mathrm{NH}\right), 7.12-7.18\left(\mathrm{~m}, 2 \mathrm{H}, \mathrm{H} 3^{\prime}\right.$ and $\mathrm{H}^{\prime}$ phenyl), 7.29-7.46 (m, 2H, H5 and H6 benzimidazole), 7.62-7.78 ( $\mathrm{m}, 2 \mathrm{H}, \mathrm{H} 2^{\prime}$ and $\mathrm{H}^{\prime}$ phenyl), 8.09-8.29 (m, 2H, H4 and $\mathrm{H} 7$ benzimidazole), 8.33 (br, $1 \mathrm{H}, \mathrm{NH}$ pyrimidine, $\mathrm{D}_{2} \mathrm{O}$ exchangeable), 11.50 (br, 2H, OH phenolic and $\mathrm{NH}$ benzimidazole, $\mathrm{D}_{2} \mathrm{O}$ exchangeable); IR ( $\left.\mathrm{KBr}, \mathrm{cm}^{-1}\right) 3347$ ( $\mathrm{NH}$ benzimidazole, NH$-\mathrm{CH}_{2}$ ), 3183 (br, NH pyrimidine, $\mathrm{H}$ bonded $\mathrm{OH}$ phenolic), 3056.6 (CH arom.), 2926 (CH aliph.), $2208(\mathrm{CN}), 1661(\mathrm{C}=\mathrm{O})$, $1610(\mathrm{C}=\mathrm{N}$ benzimidazole $), 1549,1511(\mathrm{C}=\mathrm{N}$ pyrimidine, $\mathrm{C}=$ C); MS (EI) m/z $358\left(\mathrm{M}^{+*}, 2 \%\right), 259$ (100\%). Anal. Calcd for $\mathrm{C}_{19} \mathrm{H}_{14} \mathrm{~N}_{6} \mathrm{O}_{2}: \mathrm{C}, 63.67 ; \mathrm{H}, 3.95 ; \mathrm{N}, 23.45$. Found: C, 63.95; H, $4.00 ; \mathrm{N}, 23.53$

2-((1H-Benzo[ $d]$ imidazol-2-yl)methylamino)-4-(2-chlorophenyl)-1,6-dihydro-6-oxopyrimidine-5-carbonitrile

(5e):

Yield 79\%; mp $213-215^{\circ} \mathrm{C}$ (from EtOH); ${ }^{1} \mathrm{H}-\mathrm{NMR}(200 \mathrm{MHz}$, DMSO- $\left.d_{6}\right) \delta: 5.22\left(\mathrm{~s}, 2 \mathrm{H}, \mathrm{CH}_{2}-\mathrm{NH}\right), 5.42\left(\mathrm{br}, 1 \mathrm{H}, \mathrm{CH}_{2}-\mathrm{NH}\right.$, $\mathrm{D}_{2} \mathrm{O}$ exchangeable), 7.01-7.22 (m, 2H, H5 and $\mathrm{H} 6$ benzimidazole), 7.29-7.68 (m, 6H, $\mathrm{H} 4$ and $\mathrm{H} 7$ benzimidazole, phenyl $\mathrm{H}), 8.33$ (br, $1 \mathrm{H}, \mathrm{NH}$ pyrimidine, $\mathrm{D}_{2} \mathrm{O}$ exchangeable), 13.02 (br, $1 \mathrm{H}, \mathrm{NH}$ benzimidazole, $\mathrm{D}_{2} \mathrm{O}$ exchangeable); IR ( $\mathrm{KBr}$, $\mathrm{cm}^{-1}$ ) 3401 ( $\mathrm{NH}$ benzimidazole, $\left.\underline{\mathrm{NH}}-\mathrm{CH}_{2}\right), 3151$ (NH pyrimidine), 3058 ( $\mathrm{CH}$ arom.), 2922 ( $\overline{\mathrm{CH}}$ aliph.), $2219(\mathrm{CN}), 1652$ $(\mathrm{C}=\mathrm{O}), 1623(\mathrm{C}=\mathrm{N}$ benzimidazole $), 1549,1511(\mathrm{C}=\mathrm{N}$ pyrimidine, $\mathrm{C}=\mathrm{C}$ ); $\mathrm{MS}$ (EI) $m / z$ 376/378 $\left(\mathrm{M}^{+}, 20 \% / 7 \% \mathrm{Cl}\right.$ pattern), 132 (100\%). Anal. Calcd for $\mathrm{C}_{19} \mathrm{H}_{13} \mathrm{ClN}_{6} \mathrm{O}: \mathrm{C}, 60.56 ; \mathrm{H}, 3.48$; N, 22.31. Found: C, 60.85; H, 3.64; N, 22.67.

2-((1H-Benzo[d]imidazol-2-yl)methylamino)-1,6-dihydro-4(2-hydroxyphenyl)-6-oxopyrimidine-5-carbonitrile (5f): Yield $74 \%$; mp $256-258^{\circ} \mathrm{C}$ (from $\mathrm{MeOH}$ ); ${ }^{1} \mathrm{H}-\mathrm{NMR} \quad(200 \mathrm{MHz}$, DMSO- $\left.d_{6}\right) \delta: 5.33\left(\mathrm{~s}, 2 \mathrm{H}, \underline{\mathrm{CH}}_{2}-\mathrm{NH}\right), 5.38\left(\mathrm{br}, 1 \mathrm{H}, \mathrm{CH}_{2}-\mathrm{NH}\right.$, $\mathrm{D}_{2} \mathrm{O}$ exchangeable), 7.05-7.28 ( $\mathrm{m}, 2 \mathrm{H}, \mathrm{H} 5$ and $\mathrm{H} 6$ benzimidazole), 7.30-7.65 (m, 6H, $\mathrm{H} 4$ and $\mathrm{H} 7$ benzimidazole, phenyl $\mathrm{H}), 8.33$ (br, $1 \mathrm{H}, \mathrm{NH}$ pyrimidine, $\mathrm{D}_{2} \mathrm{O}$ exchangeable), 13.14 (br, $2 \mathrm{H}, \mathrm{OH}$ phenolic and $\mathrm{NH}$ benzimidazole, $\mathrm{D}_{2} \mathrm{O}$ exchangeable); IR ( $\mathrm{KBr}, \mathrm{cm}^{-1}$ ) 3428 ( $\mathrm{NH}$ benzimidazole), 3321 (br, $\mathrm{NH}-\mathrm{CH}_{2}, \mathrm{H}$ bonded $\mathrm{OH}$ phenolic), 3108 (NH pyrimidine), $\overline{3055}$ (CH arom.), 2933 (CH aliph.), 2213 (CN), $1664(\mathrm{C}=\mathrm{O})$, $1612(\mathrm{C}=\mathrm{N}$ benzimidazole $), 1581.0,1521(\mathrm{C}=\mathrm{N}$ pyrimidine, $\mathrm{C}=\mathrm{C})$; MS (EI) m/z $357\left(\mathrm{M}^{+\cdot}, 4 \%\right), 57.27$ (100\%). Anal. Calcd for $\mathrm{C}_{19} \mathrm{H}_{14} \mathrm{~N}_{6} \mathrm{O}_{2}$ : C, 63.67; H, 3.95; N, 23.45. Found: C, 63.93; $\mathrm{H}, 3.61 ; \mathrm{N}, 23.59$.

2-((1H-Benzo[d]imidazol-2-yl)methylamino)-1,6-dihydro-6oxo-4-(thiophen-2-yl)pyrimidine-5-carbonitrile (5g): Yield $81 \%$; mp $185-187^{\circ} \mathrm{C}$ (from $\mathrm{MeOH}$ ); ${ }^{1} \mathrm{H}-\mathrm{NMR}$ (200 MHz, DMSO- $d_{6}$ ) $\delta: 4.84$ (br, $1 \mathrm{H}, \mathrm{CH}_{2}-\underline{\mathrm{NH}}, \mathrm{D}_{2} \mathrm{O}$ exchangeable), 5.40 (s, 2H, $\left.\underline{\mathrm{CH}}_{2}-\mathrm{NH}\right), 7.05-7.40(\mathrm{~m}, \overline{3 \mathrm{H}}$, thienyl $\mathrm{H}), 7.45-7.82(\mathrm{~m}$, 2H, H5 and $\mathrm{H} 6$ benzimidazole), 7.96-8.10 (m, 2H, H4 and $\mathrm{H} 7$ benzimidazole), 8.88 (br, $1 \mathrm{H}, \mathrm{NH}$ pyrimidine, $\mathrm{D}_{2} \mathrm{O}$ exchangeable), 12.96 (br, 1H, NH benzimidazole, $\mathrm{D}_{2} \mathrm{O}$ exchangeable); IR $\left(\mathrm{KBr}, \mathrm{cm}^{-1}\right) 3347$ ( $\mathrm{NH}$ benzimidazole, $\left.\underline{\mathrm{NH}}-\mathrm{CH}_{2}\right), 3152$ ( $\mathrm{NH}$ pyrimidine), 3055 (CH arom.), 2928 ( $\overline{\mathrm{CH}}$ aliph.), 2215
(CN), $1657(\mathrm{C}=\mathrm{O}), 1611(\mathrm{C}=\mathrm{N}$ benzimidazole), 1566.0, 1506 $(\mathrm{C}=\mathrm{N}$ pyrimidine, $\mathrm{C}=\mathrm{C})$; $\mathrm{MS}(\mathrm{EI}) \mathrm{m} / \mathrm{z} 348\left(\mathrm{M}^{+}, 4 \%\right), 132$ (100\%). Anal. Calcd for $\mathrm{C}_{17} \mathrm{H}_{12} \mathrm{~N}_{6} \mathrm{OS}: \mathrm{C}, 58.60 ; \mathrm{H}, 3.48 ; \mathrm{N}$, 24.13; S, 9.20. Found: C, 58.76; H, 3.75; N, 24.46; S, 9.26.

2-((1H-Benzo[ $d]$ imidazol-2-yl)methylamino)-4-(furan-2yl)-1,6-dihydro-6-oxopyrimidine-5-carbonitrile (5h): Yield $78 \%$; mp $150-152{ }^{\circ} \mathrm{C}$ (from $\mathrm{MeOH}$ ); ${ }^{1} \mathrm{H}-\mathrm{NMR}(200 \mathrm{MHz}$, DMSO- $\left.d_{6}\right) \delta: 4.90\left(\mathrm{br}, 1 \mathrm{H}, \mathrm{CH}_{2}-\underline{\mathrm{NH}}, \mathrm{D}_{2} \mathrm{O}\right.$ exchangeable), $5.88\left(\mathrm{~s}, 2 \mathrm{H}, \mathrm{CH}_{2}-\mathrm{NH}\right), 7.12-7.28(\mathrm{~m}, 3 \mathrm{H}, \mathrm{H} 5$ and $\mathrm{H} 6$ benzimidazole, $\mathrm{H}^{\prime}$ furyl), $7.56-7.64(\mathrm{~m}, 3 \mathrm{H}, \mathrm{H} 4$ and $\mathrm{H} 7$ benzimidazole, H3' furyl), 7.80-7.87 (m, 1H, H5' furyl), 8.04 (br, $1 \mathrm{H}, \mathrm{NH}$ pyrimidine, $\mathrm{D}_{2} \mathrm{O}$ exchangeable), 12.49 (br, $1 \mathrm{H}, \mathrm{NH}$ benzimidazole, $\mathrm{D}_{2} \mathrm{O}$ exchangeable); IR $\left(\mathrm{KBr}, \mathrm{cm}^{-1}\right) 3448$ ( $\mathrm{NH}$ benzimidazole), $3278\left(\mathrm{NH}-\mathrm{CH}_{2}\right), 3183$ ( $\mathrm{NH}$ pyrimidine), 3030 (CH arom.), 2950 (CH aliph.), 2203 (CN), 1684 (C=O), $1625(\mathrm{C}=\mathrm{N}$ benzimidazole $), 1591,1513(\mathrm{C}=\mathrm{N}$ pyrimidine, $\mathrm{C}=$ C); MS (EI) m/z $332\left(\mathrm{M}^{+}, 2 \%\right), 262$ (100\%). Anal. Calcd for $\mathrm{C}_{17} \mathrm{H}_{12} \mathrm{~N}_{6} \mathrm{O}_{2}$ : C, 61.44; H, 3.65; N, 25.29. Found: C, 61.23; H, $3.42 ; \mathrm{N}, 25.53$.

Preparation of 2-((1H-Benzo[d]imidazol-2-yl)methylthio or Methylamino)-1,6-dihydro-4-(aryl or heteroaryl)6-oxopyrimidine-5-carboxylic Acids (6a-h, 7a-h). General Method A solution of compounds $\mathbf{4 a}-\mathbf{h}$ or $\mathbf{5 a}-\mathbf{h}$ $(6.02 \mathrm{mmol})$ in $80 \%$ sulphuric acid $(5 \mathrm{~mL})$ was refluxed for $4 \mathrm{~h}$. The reaction mixture was cooled, filtered and the filtrate was diluted with cold water and neutralized with aqueous ammonium hydroxide. The formed precipitate was filtered, dried and recrystallized from dimethylformamide/ethanol.

2-((1H-Benzo[d]imidazol-2-yl)methylthio)-1,6-dihydro-6oxo-4-phenylpyrimidine-5-carboxylic Acid (6a): Yield 95\%; $\mathrm{mp}>300^{\circ} \mathrm{C}$ (from MeOH); ${ }^{1} \mathrm{H}-\mathrm{NMR}\left(200 \mathrm{MHz}, \mathrm{DMSO}-d_{6}\right.$ ) $\delta$ : $5.82\left(\mathrm{~s}, 2 \mathrm{H}, \mathrm{CH}_{2}-\mathrm{S}\right), 6.85\left(\mathrm{br}, 1 \mathrm{H}, \mathrm{NH}\right.$ pyrimidine, $\mathrm{D}_{2} \mathrm{O}$ exchangeable), 7.12-7.30 ( $\mathrm{m}, 2 \mathrm{H}, \mathrm{H} 5$ and $\mathrm{H} 6$ benzimidazole), 7.34-7.53 (m, 3H, H3', H4', H5' phenyl), 7.73-7.85 (m, 2H, $\mathrm{H} 2^{\prime}$ and $\mathrm{H}^{\prime}$ phenyl), 8.03-8.15 (m, 2H, H4 and $\mathrm{H} 7$ benzimidazole), 11.15 (br, 1H, COOH, $\mathrm{D}_{2} \mathrm{O}$ exchangeable), 11.71 (br, $1 \mathrm{H}, \mathrm{NH}$ benzimidazole, $\mathrm{D}_{2} \mathrm{O}$ exchangeable); IR $\left(\mathrm{KBr}, \mathrm{cm}^{-1}\right)$ 3417 ( $\mathrm{NH}$ benzimidazole), 3127 (br, $\mathrm{H}$ bonded $\mathrm{OH}$ carboxylic, NH pyrimidine), 3050 (CH arom.), 2937 (CH aliph.), 1705 ( $\mathrm{C}=\mathrm{O}$ carboxylic), $1661(\mathrm{C}=\mathrm{O}$ amide $), 1620(\mathrm{C}=\mathrm{N}$ benzimidazole), 1509, $1560(\mathrm{C}=\mathrm{N}$ pyrimidine, $\mathrm{C}=\mathrm{C})$. Anal. Calcd for $\mathrm{C}_{19} \mathrm{H}_{14} \mathrm{~N}_{4} \mathrm{O}_{3} \mathrm{~S}: \mathrm{C}, 60.31 ; \mathrm{H}, 3.74 ; \mathrm{N}, 14.81 ; \mathrm{S}, 8.47$. Found: C, $60.65 ; \mathrm{H}, 4.00 ; \mathrm{N}, 15.02 ; \mathrm{S}, 8.78$.

2-((1H-Benzo[d]imidazol-2-yl)methylthio)-4-(4-chlorophenyl)-1,6-dihydro-6-oxopyrimidine-5-carboxylic Acid (6b): Yield $90 \%$; mp $>300^{\circ} \mathrm{C}$ (from $\mathrm{MeOH}$ ); ${ }^{1} \mathrm{H}-\mathrm{NMR}(200 \mathrm{MHz}$, DMSO- $\left.d_{6}\right) \delta: 5.83$ (s, 2H, $\left.\mathrm{CH}_{2}-\mathrm{S}\right), 7.22$ (br, $1 \mathrm{H}, \mathrm{NH}$ pyrimidine, $\mathrm{D}_{2} \mathrm{O}$ exchangeable), 7.54-7.57 ( $\mathrm{m}, 4 \mathrm{H}, \mathrm{H} 5$ and $\mathrm{H} 6$ benzimidazole, $\mathrm{H}^{\prime}$ and $\mathrm{H}^{\prime}$ phenyl), 7.73-7.76 (m, 4H, H4 and $\mathrm{H} 7$ benzimidazole, $\mathrm{H}_{2}^{\prime}$ and $\mathrm{H}^{\prime}$ phenyl), 11.15 (br, $2 \mathrm{H}, \mathrm{COOH}$ and $\mathrm{NH}$ benzimidazole, $\mathrm{D}_{2} \mathrm{O}$ exchangeable); ${ }^{13} \mathrm{C}-\mathrm{NMR}(\overline{70 \mathrm{MHz}}$, DMSO- $\left.d_{6}\right) \delta: 27.0\left(\mathrm{CH}_{2}\right), 119.0-139.0(\mathrm{Ar}-\mathrm{C}), 149.0-154.0(\mathrm{C}=$ $\mathrm{N}$ groups), 162.5 ( $\mathrm{C}=\mathrm{O}$ amide), $168.0(\mathrm{C}=\mathrm{O}$ carboxylic); IR $\left(\mathrm{KBr}, \mathrm{cm}^{-1}\right) 3419$ (NH benzimidazole), 3281 (br, H bonded $\mathrm{OH}$ carboxylic), 3179 ( $\mathrm{NH}$ pyrimidine), 3055 (CH arom.), 2960 (CH aliph.), 1702 ( $\mathrm{C}=\mathrm{O}$ carboxylic), 1654 ( $\mathrm{C}=\mathrm{O}$ amide), 1624 $(\mathrm{C}=\mathrm{N}$ benzimidazole), 1541, $1521(\mathrm{C}=\mathrm{N}$ pyrimidine, $\mathrm{C}=\mathrm{C})$. Anal. Calcd for $\mathrm{C}_{19} \mathrm{H}_{13} \mathrm{ClN}_{4} \mathrm{O}_{3} \mathrm{~S}: \mathrm{C}, 55.27 ; \mathrm{H}, 3.18 ; \mathrm{N}, 13.57$; , 7.77. Found: C, 55.56; H, 3.52; N, 13.86; S, 7.90.

2-((1H-Benzo[d]imidazol-2-yl)methylamino)-4-(4-fluorophenyl)-1,6-dihydro-6-oxopyrimidine-5-carboxylic Acid (6c): 
Yield 91\%; mp $>300^{\circ} \mathrm{C}$ (from $\mathrm{MeOH}$ ); ${ }^{1} \mathrm{H}-\mathrm{NMR}(200 \mathrm{MHz}$, DMSO- $\left.d_{6}\right) \delta: 5.53\left(\mathrm{~s}, 2 \mathrm{H}, \mathrm{CH}_{2}-\mathrm{S}\right), 7.28(\mathrm{br}, 1 \mathrm{H}, \mathrm{NH}$ pyrimidine, $\mathrm{D}_{2} \mathrm{O}$ exchangeable), 7.42-7.53 (m, 4H, H5 and $\mathrm{H} 6$ benzimidazole, $\mathrm{H}^{\prime}$ and $\mathrm{H}^{\prime}$ phenyl), 7.66-7.73 (m, 4H, H4 and $\mathrm{H} 7$ benzimidazole, $\mathrm{H} 2^{\prime}$ and $\mathrm{H}^{\prime}$ phenyl), 11.22 (br, $2 \mathrm{H}$, $\mathrm{COOH}$ and $\mathrm{NH}$ benzimidazole, $\mathrm{D}_{2} \mathrm{O}$ exchangeable); IR ( $\mathrm{KBr}$, $\mathrm{cm}^{-1}$ ) 3406 (NH benzimidazole), 3216 (br, $\mathrm{H}$ bonded $\mathrm{OH}$ carboxylic), 3109 ( $\mathrm{NH}$ pyrimidine), 3055 (CH arom.), 2923 (CH aliph.), 1701 ( $\mathrm{C}=\mathrm{O}$ carboxylic), 1654 ( $\mathrm{C}=\mathrm{O}$ amide), $1626(\mathrm{C}=$ $\mathrm{N}$ benzimidazole), 1562, $1530(\mathrm{C}=\mathrm{N}$ pyrimidine, $\mathrm{C}=\mathrm{C})$. Anal. Calcd for $\mathrm{C}_{19} \mathrm{H}_{13} \mathrm{FN}_{4} \mathrm{O}_{3} \mathrm{~S}$ : C, 57.57; H, 3.31; N, 14.14; S, 8.09. Found: C, 57.96; H, 3.56; N, 14.47; S, 8.32.

2-((1H-Benzo[d]imidazol-2-yl)methylthio)-1,6-dihydro-4(4-hydroxyphenyl)-6-oxopyrimidine-5-carboxylic Acid (6d): Yield $96 \%$; mp $>300^{\circ} \mathrm{C}$ (from $\mathrm{MeOH}$ ); ${ }^{1} \mathrm{H}-\mathrm{NMR}(200 \mathrm{MHz}$, DMSO- $\left.d_{6}\right) \delta: 5.50\left(\mathrm{~s}, 2 \mathrm{H}, \underline{\mathrm{CH}}_{2}-\mathrm{S}\right), 7.21-7.29$ (m, 2H, H3' and H5' phenyl), 7.34-7.45 (m, 2H, H5 and H6 benzimidazole), 7.52-7.58 (m, 2H, H2' and $\mathrm{H}^{\prime}$ phenyl), 8.12-8.22 (m, 2H, H4 and $\mathrm{H} 7$ benzimidazole), $8.35\left(\mathrm{br}, 1 \mathrm{H}, \mathrm{NH}\right.$ pyrimidine, $\mathrm{D}_{2} \mathrm{O}$ exchangeable), 10.50 (s, 1H, OH phenolic $\mathrm{D}_{2} \mathrm{O}$ exchangeable); 11.15 (br, $2 \mathrm{H}, \mathrm{COOH}$ and $\mathrm{NH}$ benzimidazole, $\mathrm{D}_{2} \mathrm{O}$ exchangeable); IR ( $\mathrm{KBr}, \overline{\mathrm{cm}^{-1}}$ ) 3400 ( $\mathrm{NH}$ benzimidazole), 3184 (br, $\mathrm{H}$ bonded $\mathrm{OH}$ carboxylic), 3109 ( $\mathrm{NH}$ pyrimidine), $3055(\mathrm{CH}$ arom.), 2936 (CH aliph.), 1701 ( $\mathrm{C}=\mathrm{O}$ carboxylic), $1654(\mathrm{C}=\mathrm{O}$ amide $), 1627(\mathrm{C}=\mathrm{N}$ benzimidazole $), 1560,1540(\mathrm{C}=\mathrm{N}$ pyrimidine, $\mathrm{C}=\mathrm{C})$. Anal. Calcd for $\mathrm{C}_{19} \mathrm{H}_{14} \mathrm{~N}_{4} \mathrm{O}_{4} \mathrm{~S}$ : C, 57.86; H, 3.58; N, 14.21; S, 8.13. Found: C, 58.00; H, 3.62; N, 14.52; S, 8.45.

2-((1H-Benzo[d]imidazol-2-yl)methylthio)-4-(2-chlorophenyl)-1,6-dihydro-6-oxopyrimidine-5-carboxylic Acid (6e): Yield 93\%; mp $>300{ }^{\circ} \mathrm{C}$ (from $\mathrm{MeOH}$ ); ${ }^{1} \mathrm{H}-\mathrm{NMR}(200 \mathrm{MHz}$, DMSO- $\left.d_{6}\right) \delta: 5.36\left(\mathrm{~s}, 2 \mathrm{H}, \mathrm{CH}_{2}-\mathrm{S}\right), 7.29-7.38(\mathrm{~m}, 2 \mathrm{H}, \mathrm{H} 5$ and $\mathrm{H} 6$ benzimidazole), 7.83-7.90 ( $\mathrm{m}, 6 \mathrm{H}, \mathrm{H} 4$ and $\mathrm{H} 7$ benzimidazole, 4 phenyl $\mathrm{H}$ ), 8.10 (br, $1 \mathrm{H}, \mathrm{NH}$ pyrimidine, $\mathrm{D}_{2} \mathrm{O}$ exchangeable), 9.90 (br, $1 \mathrm{H}, \mathrm{COOH}, \mathrm{D}_{2} \mathrm{O}$ exchangeable), 12.50 (br, $1 \mathrm{H}, \mathrm{NH}$ benzimidazole, $\overline{\mathrm{D}_{2} \mathrm{O}}$ exchangeable); IR ( $\mathrm{KBr}$, $\mathrm{cm}^{-1}$ ) 3379 ( $\mathrm{NH}$ benzimidazole), 3230 (br, $\mathrm{H}$ bonded $\mathrm{OH}$ carboxylic), 3174 (NH pyrimidine), 3054 (CH arom.), 2930 (CH aliph.), 1707 ( $\mathrm{C}=\mathrm{O}$ carboxylic), 1664 ( $\mathrm{C}=\mathrm{O}$ amide), 1624 (C= $\mathrm{N}$ benzimidazole $), 1560,1542(\mathrm{C}=\mathrm{N}$ pyrimidine, $\mathrm{C}=\mathrm{C})$. Anal. Calcd for $\mathrm{C}_{19} \mathrm{H}_{13} \mathrm{ClN}_{4} \mathrm{O}_{3} \mathrm{~S}$ : C, 55.27; H, 3.18; N, 13.57; S, 7.77. Found: C, 55.55; H, 3.42; N, 13.77; S, 7.80.

2-((1H-Benzo[d]imidazol-2-yl)methylthio)-1,6-dihydro-4(2-hydroxyphenyl)-6-oxopyrimidine-5-carboxylic Acid (6f): Yield $91 \%$; mp $>300^{\circ} \mathrm{C}$ (from $\mathrm{MeOH}$ ); ${ }^{1} \mathrm{H}-\mathrm{NMR}(200 \mathrm{MHz}$, DMSO- $\left.d_{6}\right) \delta: 5.38\left(\mathrm{~s}, 2 \mathrm{H}, \mathrm{CH}_{2}-\mathrm{S}\right), 7.08-7.62(\mathrm{~m}, 8 \mathrm{H}, \mathrm{Ar}-\mathrm{H})$, 8.81 (br, $2 \mathrm{H}, \mathrm{OH}$ phenolic and $\mathrm{NH}$ pyrimidine, $\mathrm{D}_{2} \mathrm{O}$ exchangeable), 9.04 (br, $2 \mathrm{H}, \mathrm{COOH}$ and $\mathrm{NH}$ benzimidazole, $\mathrm{D}_{2} \mathrm{O}$ exchangeable); IR ( $\mathrm{KBr}, \mathrm{cm}^{-1}$ ) 3390 ( $\mathrm{NH}$ benzimidazole), 3212 (br, $\mathrm{H}$ bonded $\mathrm{OH}$ carboxylic and $\mathrm{OH}$ phenolic, $\mathrm{NH}$ pyrimidine), 3056 ( $\mathrm{CH}$ arom.), 2920 ( $\mathrm{CH}$ aliph.), 1702 (C=O carboxylic), 1650 ( $\mathrm{C}=\mathrm{O}$ amide), 1609 ( $\mathrm{C}=\mathrm{N}$ benzimidazole), 1562, $1540(\mathrm{C}=\mathrm{N}$ pyrimidine, $\mathrm{C}=\mathrm{C})$. Anal. Calcd for $\mathrm{C}_{19} \mathrm{H}_{14} \mathrm{~N}_{4} \mathrm{O}_{4} \mathrm{~S}$ : C, 57.86; H, 3.58; N, 14.21; S, 8.13. Found: C, 57.66; H, 3.74; $\mathrm{N}, 14.52 ; \mathrm{S}, 8.32$.

2-((1H-Benzo[d]imidazol-2-yl)methylthio)-1,6-dihydro-6oxo-4-(thiophen-2-yl)pyrimidine-5-carboxylic Acid (6g): Yield $93 \% ; \quad \mathrm{mp}>300^{\circ} \mathrm{C}$ (from $\mathrm{MeOH}$ ); ${ }^{1} \mathrm{H}-\mathrm{NMR} \quad(200 \mathrm{MHz}$, DMSO-d $d_{6} \delta: 5.84\left(\mathrm{~s}, 2 \mathrm{H}, \underline{\mathrm{CH}}_{2}-\mathrm{S}\right), 7.15-7.42$ (m, 3H, thienyl $\mathrm{H}), 7.46-7.83$ (m, 2H, H5 and H6 benzimidazole), 7.89-8.12 ( $\mathrm{m}, 2 \mathrm{H}, \mathrm{H} 4$ and $\mathrm{H} 7$ benzimidazole), 8.58 (br, 1H, NH pyrimidine, $\mathrm{D}_{2} \mathrm{O}$ exchangeable), 12.94 (br, $2 \mathrm{H}, \mathrm{COOH}$ and $\mathrm{NH}$ benzimidazole, $\mathrm{D}_{2} \mathrm{O}$ exchangeable); IR $\left(\mathrm{KBr}, \mathrm{cm}^{-1}\right) 3406(\mathrm{NH}$ benzimidazole), 3216 (br, $\mathrm{H}$ bonded $\mathrm{OH}$ carboxylic), 3109 (NH pyrimidine), 3055 ( $\mathrm{CH}$ arom.), 2923 (CH aliph.), 1701 $(\mathrm{C}=\mathrm{O}$ carboxylic), $1654(\mathrm{C}=\mathrm{O}$ amide $), 1626(\mathrm{C}=\mathrm{N}$ benzimidazole), 1562, $1530(\mathrm{C}=\mathrm{N}$ pyrimidine, $\mathrm{C}=\mathrm{C})$. Anal. Calcd for $\mathrm{C}_{17} \mathrm{H}_{12} \mathrm{~N}_{4} \mathrm{O}_{3} \mathrm{~S}_{2}$ : C, 53.11; H, 3.15; N, 14.58; S, 16.68. Found: C, 53.42; H, 3.35; N, 14.75; S, 16.23.

2-((1H-Benzo[d]imidazol-2-yl)methylthio)-4-(furan-2yl)-1,6-dihydro-6-oxopyrimidine-5-carboxylic Acid (6h): Yield $94 \%$; mp $>300^{\circ} \mathrm{C}$ (from $\mathrm{MeOH}$ ); ${ }^{1} \mathrm{H}-\mathrm{NMR}(200 \mathrm{MHz}, \mathrm{DMSO}-$ $\left.d_{6}\right) \delta: 5.23\left(\mathrm{~s}, 2 \mathrm{H}, \mathrm{CH}_{2}-\mathrm{S}\right), 5.79-5.88\left(\mathrm{~m}, 1 \mathrm{H}, \mathrm{H}^{\prime}\right.$ furyl $)$, 7.13-7.31 (m, 3H, H5 and $\mathrm{H} 6$ benzimidazole, H3' furyl), 7.54-7.63 (m, 3H, H4 and $\mathrm{H} 7$ benzimidazole, $\mathrm{H}^{\prime}$ furyl), 8.00 (br, $1 \mathrm{H}, \mathrm{NH}$ pyrimidine, $\mathrm{D}_{2} \mathrm{O}$ exchangeable), 12.40 (br, $2 \mathrm{H}$, $\mathrm{COOH}$ and $\mathrm{NH}$ benzimidazole, $\mathrm{D}_{2} \mathrm{O}$ exchangeable); IR ( $\mathrm{KBr}$, $\mathrm{cm}^{-1}$ ) 3388 ( $\mathrm{NH}$ benzimidazole), 3176 (br, $\mathrm{H}$ bonded $\mathrm{OH}$ carboxylic, NH pyrimidine), 3056 ( $\mathrm{CH}$ arom.), 2920 (CH aliph.), $1700(\mathrm{C}=\mathrm{O}$ carboxylic), $1654(\mathrm{C}=\mathrm{O}$ amide $), 1617(\mathrm{C}=\mathrm{N}$ benzimidazole), 1580, $1532(\mathrm{C}=\mathrm{N}$ pyrimidine, $\mathrm{C}=\mathrm{C})$. Anal. Calcd for $\mathrm{C}_{17} \mathrm{H}_{12} \mathrm{~N}_{4} \mathrm{O}_{4} \mathrm{~S}$ : C, 55.43; H, 3.29; N, 15.21; S, 8.70. Found: C, 55.80; H, 3.19; N, 15.52; S, 8.98.

2-((1H-Benzo[d]imidazol-2-yl)methylamino)-1,6-dihydro-6oxo-4-phenylpyrimidine-5-carboxylic Acid (7a): Yield 94\%; $\mathrm{mp}>300^{\circ} \mathrm{C}$ (from MeOH); ${ }^{1} \mathrm{H}-\mathrm{NMR}\left(200 \mathrm{MHz}, \mathrm{DMSO}-d_{6}\right) \delta$ : 5.07 (s, $2 \mathrm{H}, \underline{\mathrm{CH}}_{2}-\mathrm{NH}$ ), 6.31 (br, $1 \mathrm{H}, \mathrm{CH}_{2}-\mathrm{NH}, \mathrm{D}_{2} \mathrm{O}$ exchangeable), $7.40-8.35$ (m, 9H, Ar-H), 9.72 (br, $1 \mathrm{H}, \mathrm{NH}$ pyrimidine, $\mathrm{D}_{2} \mathrm{O}$ exchangeable), 10.83 (br, $1 \mathrm{H}, \mathrm{COOH}, \mathrm{D}_{2} \mathrm{O}$ exchangeable), 11.85 (br, $1 \mathrm{H}, \mathrm{NH}$ benzimidazole, $\widehat{\mathrm{D}_{2} \mathrm{O}}$ exchangeable); IR $\left(\mathrm{KBr}, \mathrm{cm}^{-1}\right) 3406$ (NH benzimidazole), 3219 (br, H bonded $\mathrm{OH}$ carboxylic, $\left.\mathrm{NH}-\mathrm{CH}_{2}\right), 3117(\mathrm{NH}$ pyrimidine), $3053(\mathrm{CH}$ arom.), 2990 ( $\mathrm{CH}$ aliph.), $1700(\mathrm{C}=\mathrm{O}$ carboxylic), $1651(\mathrm{C}=\mathrm{O}$ amide), 1619 ( $\mathrm{C}=\mathrm{N}$ benzimidazole $), 1559,1521(\mathrm{C}=\mathrm{N}$ pyrimidine, $\mathrm{C}=\mathrm{C}$ ). Anal. Calcd for $\mathrm{C}_{19} \mathrm{H}_{15} \mathrm{~N}_{5} \mathrm{O}_{3}: \mathrm{C}, 63.15 ; \mathrm{H}, 4.19 ; \mathrm{N}$, 19.38. Found: C, 63.45; H, 4.32; N, 19.55 .

2-((1H-Benzo[d]imidazol-2-yl)methylamino)-4-(4-chlorophenyl)-1,6-dihydro-6-oxopyrimidine-5-carboxylic Acid (7b): Yield 93\%; mp $>300^{\circ} \mathrm{C}$ (from $\mathrm{MeOH}$ ); ${ }^{1} \mathrm{H}-\mathrm{NMR}(200 \mathrm{MHz}$, DMSO- $\left.d_{6}\right) \delta: 5.74\left(\mathrm{~s}, 2 \mathrm{H}, \underline{\mathrm{CH}}_{2}-\mathrm{NH}\right), 6.13$ (br, $1 \mathrm{H}, \mathrm{CH}_{2}-\mathrm{NH}$, $\mathrm{D}_{2} \mathrm{O}$ exchangeable), 7.09-7.25 (m, 2H, H5 and H6 benzimidazole), 7.37 (d, $J=8 \mathrm{~Hz}, 2 \mathrm{H}, \mathrm{H} 3^{\prime}$ and $\mathrm{H} 5^{\prime}$ phenyl), 7.51-7.62 (m, 2H, H4 and $\mathrm{H} 7$ benzimidazole), 7.77 (d, $J=8 \mathrm{~Hz}, 2 \mathrm{H}, \mathrm{H} 2^{\prime}$ and $\mathrm{H}^{\prime}$ phenyl), 8.00 (br, $1 \mathrm{H}, \mathrm{NH}$ pyrimidine, $\mathrm{D}_{2} \mathrm{O}$ exchangeable), 10.13 (br, $1 \mathrm{H}, \mathrm{COOH}, \mathrm{D}_{2} \mathrm{O}$ exchangeable), 12.60 (br, $1 \mathrm{H}, \mathrm{NH}$ benzimidazole, $\overline{\mathrm{D}_{2} \mathrm{O}}$ exchangeable); IR $\left(\mathrm{KBr}, \mathrm{cm}^{-1}\right)$ 3406 ( $\mathrm{NH}$ benzimidazole), 3222 (br, $\mathrm{H}$ bonded $\mathrm{OH}$ carboxylic, $\left.\mathrm{NH}-\mathrm{CH}_{2}\right), 3109$ ( $\mathrm{NH}$ pyrimidine), $3056(\mathrm{CH}$ arom.), $2920(\mathrm{CH}$ aliph.), 1715 ( $\mathrm{C}=\mathrm{O}$ carboxylic), 1650 ( $\mathrm{C}=\mathrm{O}$ amide), 1609 ( $\mathrm{C}=$ $\mathrm{N}$ benzimidazole), 1559, $1539(\mathrm{C}=\mathrm{N}$ pyrimidine, $\mathrm{C}=\mathrm{C})$. Anal. Calcd for $\mathrm{C}_{19} \mathrm{H}_{14} \mathrm{ClN}_{5} \mathrm{O}_{3}: \mathrm{C}, 57.65 ; \mathrm{H}, 3.57 ; \mathrm{N}, 17.70$. Found: $\mathrm{C}$, 57.43; H, 3.67; N, 17.86.

2-((1H-Benzo[d]imidazol-2-yl)methylamino)-4-(4-fluorophenyl)-1,6-dihydro-6-oxopyrimidine-5-carboxylic Acid (7c): Yield 93\%; mp $>300^{\circ} \mathrm{C}$ (from $\mathrm{MeOH}$ ); ${ }^{1} \mathrm{H}-\mathrm{NMR}(200 \mathrm{MHz}$, DMSO- $\left.d_{6}\right) \delta: 5.81\left(\mathrm{~s}, 2 \mathrm{H}, \underline{\mathrm{CH}}_{2}-\mathrm{NH}\right), 6.85$ (br, $1 \mathrm{H}, \mathrm{CH}_{2}-\underline{\mathrm{NH}}$, $\mathrm{D}_{2} \mathrm{O}$ exchangeable), $7.10\left(\mathrm{br}, 1 \mathrm{H}, \mathrm{NH}\right.$ pyrimidine, $\mathrm{D}_{2} \mathrm{O}$ exchangeable), 7.30-7.39 (m, 4H, H5 and H6 benzimidazole, H3' and $\mathrm{H} 5^{\prime}$ phenyl), 7.77-7.84 (m, 4H, $\mathrm{H} 4$ and $\mathrm{H} 7$ benzimidazole, $\mathrm{H} 2^{\prime}$ and $\mathrm{H6}^{\prime}$ phenyl), 11.12 (br, 2H, $\mathrm{COOH}$ and $\mathrm{NH}$ benzimidazole, $\mathrm{D}_{2} \mathrm{O}$ exchangeable); ${ }^{13} \mathrm{C}-\mathrm{NMR}(70 \mathrm{MHz}$, DMSO$\left.d_{6}\right) \delta: 27.0\left(\mathrm{CH}_{2}\right), 114.0-137.0(\mathrm{Ar}-\mathrm{C}), 151.0-165.0(\mathrm{C}-\mathrm{F}, \mathrm{C}=$ $\mathrm{N}$ groups), $166.0(\mathrm{C}=\mathrm{O}$ amide $), 167.5(\mathrm{C}=\mathrm{O}$ carboxylic); IR 
$\left(\mathrm{KBr}, \mathrm{cm}^{-1}\right) 3420$ ( $\mathrm{NH}$ benzimidazole), 3166 (br, $\mathrm{H}$ bonded $\mathrm{OH}$ carboxylic, $\mathrm{NH}-\mathrm{CH}_{2}, \mathrm{NH}$ pyrimidine), 3033 ( $\mathrm{CH}$ arom.), 2982 ( $\mathrm{CH}$ aliph.), 1713 ( $\mathrm{C}=\mathrm{O}$ carboxylic), 1668 ( $\mathrm{C}=\mathrm{O}$ amide), $1620(\mathrm{C}=\mathrm{N}$ benzimidazole $), 1543,1512(\mathrm{C}=\mathrm{N}$ pyrimidine, $\mathrm{C}=$ C). Anal. Calcd for $\mathrm{C}_{19} \mathrm{H}_{14} \mathrm{FN}_{5} \mathrm{O}_{3}: \mathrm{C}, 60.16 ; \mathrm{H}, 3.73 ; \mathrm{N}, 18.47$. Found: C, 60.45; H, 3.86; N, 18.67 .

2-((1H-Benzo[d]imidazol-2-yl)methyamino)-1,6-dihydro-4(4-hydroxyphenyl)-6-oxopyrimidine-5-carboxylic Acid (7d): Yield $96 \%$; mp $>300^{\circ} \mathrm{C}$ (from $\mathrm{MeOH}$ ); ${ }^{1} \mathrm{H}-\mathrm{NMR}(200 \mathrm{MHz}$, DMSO- $d_{6}$ ) $\delta: 5.23$ (br, $1 \mathrm{H}, \mathrm{CH}_{2}-\underline{\mathrm{NH}}, \mathrm{D}_{2} \mathrm{O}$ exchangeable), 5.50 (s, 2H, $\underline{\mathrm{CH}}_{2}-\mathrm{NH}$ ), 7.21-7.29 (m, 2H, H3' and $\mathrm{H}^{\prime}$ phenyl), 7.34-7.45 (m, 2H, H5 and H6 benzimidazole), 7.52-7.58 (m, $2 \mathrm{H}, \mathrm{H} 2^{\prime}$ and $\mathrm{H}^{\prime}$ phenyl), 8.12-8.22 (m, 2H, H4 and $\mathrm{H} 7$ benzimidazole), 8.35 (br, $1 \mathrm{H}, \mathrm{NH}$ pyrimidine, $\mathrm{D}_{2} \mathrm{O}$ exchangeable), $10.50\left(\mathrm{~s}, 1 \mathrm{H}, \mathrm{OH}\right.$ phenolic $\mathrm{D}_{2} \mathrm{O}$ exchangeable); $11.15(\mathrm{br}, 2 \mathrm{H}$, $\mathrm{COOH}$ and $\mathrm{NH}$ benzimidazole, $\mathrm{D}_{2} \mathrm{O}$ exchangeable); IR ( $\mathrm{KBr}$, $\mathrm{cm}^{-1}$ ) 3425 ( $\mathrm{NH}$ benzimidazole), 3166 (br, $\mathrm{H}$ bonded $\mathrm{OH}$ carboxylic and $\mathrm{OH}$ phenolic, $\mathrm{NH}-\mathrm{CH}_{2}, \mathrm{NH}$ pyrimidine), 3056 (CH arom.), 2923 (CH aliph.), 1702 (C=O carboxylic), 1650 $(\mathrm{C}=\mathrm{O}$ amide $), 1611(\mathrm{C}=\mathrm{N}$ benzimidazole $), 1546,1520(\mathrm{C}=\mathrm{N}$ pyrimidine, $\mathrm{C}=\mathrm{C}$ ). Anal. Calcd for $\mathrm{C}_{19} \mathrm{H}_{15} \mathrm{~N}_{5} \mathrm{O}_{4}: \mathrm{C}, 60.47 ; \mathrm{H}$, 4.01; N, 18.56. Found: C, 60.64; H, 3.95; N, 18.75.

2-((1H-Benzo[d]imidazol-2-yl)methylamino)-4-(2-chlorophenyl)-1,6-dihydro-6-oxopyrimidine-5-carboxylic Acid (7e): Yield $96 \%$; mp $>300{ }^{\circ} \mathrm{C}$ (from $\mathrm{MeOH}$ ); ${ }^{1} \mathrm{H}-\mathrm{NMR}(200 \mathrm{MHz}$, DMSO- $\left.d_{6}\right) \delta: 4.90\left(\mathrm{br}, 1 \mathrm{H}, \mathrm{CH}_{2}-\mathrm{NH}, \mathrm{D}_{2} \mathrm{O}\right.$ exchangeable), 5.50 (s, 2H, $\left.\underline{\mathrm{CH}}_{2}-\mathrm{NH}\right), 7.19-7.41(\mathrm{~m}, 2 \mathrm{H}, \mathrm{H} 5$ and $\mathrm{H} 6$ benzimidazole), 7.54-7.79 (m, 6H, H4 and $\mathrm{H} 7$ benzimidazole and 4 phenyl $\mathrm{H}$ ), 9.40 (br, $1 \mathrm{H}, \mathrm{NH}$ pyrimidine, $\mathrm{D}_{2} \mathrm{O}$ exchangeable), 10.50 (br, $2 \mathrm{H}, \mathrm{COOH}$ and $\mathrm{NH}$ benzimidazole, $\mathrm{D}_{2} \mathrm{O}$ exchangeable); IR ( $\mathrm{KBr}, \mathrm{cm}^{-1}$ ) 3390 ( $\mathrm{NH}$ benzimidazole), 3216 (br, $\mathrm{H}$ bonded $\mathrm{OH}$ carboxylic, $\mathrm{NH}-\mathrm{CH}_{2}$ ), 3125 ( $\mathrm{NH}$ pyrimidine), 3053 ( $\mathrm{CH}$ arom.), 2930 ( $\mathrm{C} \overline{\mathrm{H}}$ aliph.), 1700 ( $\mathrm{C}=\mathrm{O}$ carboxylic), $1651(\mathrm{C}=\mathrm{O}$ amide), $1619(\mathrm{C}=\mathrm{N}$ benzimidazole), 1560, 1538 $(\mathrm{C}=\mathrm{N}$ pyrimidine, $\mathrm{C}=\mathrm{C})$. Anal. Calcd for $\mathrm{C}_{19} \mathrm{H}_{14} \mathrm{ClN}_{5} \mathrm{O}_{3}: \mathrm{C}$, 57.65; H, 3.57; N, 17.70. Found: C, 57.78; H, 3.77; N, 17.57.

2-((1H-Benzo[d]imidazol-2-yl)methylamino)-1,6-dihydro-4(2-hydroxyphenyl)-6-oxopyrimidine-5-carboxylic Acid (7f): Yield $90 \%$; mp $>300^{\circ} \mathrm{C}$ (from $\mathrm{MeOH}$ ); ${ }^{1} \mathrm{H}-\mathrm{NMR}(200 \mathrm{MHz}$, DMSO- $\left.d_{6}\right) \delta: 5.01$ (br, $1 \mathrm{H}, \mathrm{CH}_{2}-\underline{\mathrm{NH}}, \mathrm{D}_{2} \mathrm{O}$ exchangeable), 5.30 (s, 2H, $\left.\underline{\mathrm{CH}}_{2}-\mathrm{NH}\right), 7.60-7.87(\mathrm{~m}, \overline{8 \mathrm{H}}, \mathrm{Ar}-\mathrm{H}), 8.01$ (br, $1 \mathrm{H}, \mathrm{NH}$ pyrimidine, $\mathrm{D}_{2} \mathrm{O}$ exchangeable), 10.22 (s., $1 \mathrm{H}, \mathrm{OH}$ phenolic, $\mathrm{D}_{2} \mathrm{O}$ exchangeable), 11.82 (br, $1 \mathrm{H}, \mathrm{COOH}, \mathrm{D}_{2} \mathrm{O}$ exchangeable), 13.32 (br, $1 \mathrm{H}, \mathrm{NH}$ benzimidazole, $\overline{\mathrm{D}_{2} \mathrm{O}}$ exchangeable); IR $\left(\mathrm{KBr}, \mathrm{cm}^{-1}\right) 3406$ ( $\mathrm{NH}$ benzimidazole), 3225 (br, $\mathrm{H}$ bonded $\mathrm{OH}$ carboxylic and $\mathrm{OH}$ phenolic, $\left.\mathrm{NH}-\mathrm{CH}_{2}\right), 3056$ ( $\mathrm{CH}$ arom.), 2957 ( $\mathrm{CH}$ aliph.), 1719 ( $\mathrm{C}=\mathrm{O}$ carboxylic), 1651 ( $\mathrm{C}=\mathrm{O}$ amide), $1617(\mathrm{C}=\mathrm{N}$ benzimidazole $), 1559,1533(\mathrm{C}=\mathrm{N}$ pyrimidine, $\mathrm{C}=$ C). Anal. Calcd for $\mathrm{C}_{19} \mathrm{H}_{15} \mathrm{~N}_{5} \mathrm{O}_{4}: \mathrm{C}, 60.47 ; \mathrm{H}, 4.01 ; \mathrm{N}, 18.56$. Found: C, 60.27; H, 3.75; N, 18.65.

2-((1H-Benzo[d]imidazol-2-yl)methylamino)-1,6-dihydro-6oxo-4-(thiophen-2-yl)pyrimidine-5-carboxylic Acid (7g): Yield $91 \%$; mp $>300^{\circ} \mathrm{C}$ (from $\mathrm{MeOH}$ ); ${ }^{1} \mathrm{H}-\mathrm{NMR}$ (200 MHz, DMSO$\left.d_{6}\right) \delta: 5.19$ (br, $1 \mathrm{H}, \mathrm{CH}_{2}-\underline{\mathrm{NH}}, \mathrm{D}_{2} \mathrm{O}$ exchangeable), 5.79 (s, 2H, $\left.\underline{\mathrm{CH}}_{2}-\mathrm{NH}\right), 7.18-7.37(\mathrm{~m}, 3 \mathrm{H}$, thienyl H), 7.55-7.65 (m, 2H, H5 and $\mathrm{H} 6$ benzimidazole), 7.95-8.12 ( $\mathrm{m}, 2 \mathrm{H}, \mathrm{H} 4$ and $\mathrm{H} 7$ benzimidazole), 8.85 (br, $1 \mathrm{H}, \mathrm{NH}$ pyrimidine, $\mathrm{D}_{2} \mathrm{O}$ exchangeable), 12.45 (br, $1 \mathrm{H}, \mathrm{COOH}, \mathrm{D}_{2} \mathrm{O}$ exchangeable), 12.90 (br, $1 \mathrm{H}, \mathrm{NH}$ benzimidazole, $\mathrm{D}_{2} \overline{\mathrm{O}}$ exchangeable); IR $\left(\mathrm{KBr}, \mathrm{cm}^{-1}\right) 3375(\mathrm{NH}$ benzimidazole), 3170 (br, $\mathrm{H}$ bonded $\mathrm{OH}$ carboxylic, $\mathrm{NH}-\mathrm{CH}_{2}$, NH pyrimidine), 3052 (Ar-H), 2930 (CH aliph.), 1705 (C=
O carboxylic), $1659(\mathrm{C}=\mathrm{O}$ amide $), 1624(\mathrm{C}=\mathrm{N}$ benzimidazole) 1560, $1542(\mathrm{C}=\mathrm{N}$ pyrimidine, $\mathrm{C}=\mathrm{C})$. Anal. Calcd for $\mathrm{C}_{17} \mathrm{H}_{13} \mathrm{~N}_{5} \mathrm{O}_{3} \mathrm{~S}: \mathrm{C}, 55.58 ; \mathrm{H}, 3.57 ; \mathrm{N}, 19.07 ; \mathrm{S}, 8.73$. Found: C, 55.86; H, 3.76; N, 19.35; S, 8.84.

2-((1H-Benzo[ $d]$ imidazol-2-yl)methylamino)-4-(furan-2yl)-1,6-dihydro-6-oxopyrimidine-5-carboxylic Acid (7h): Yield $92 \%$; mp $>300^{\circ} \mathrm{C}$ (from MeOH); ${ }^{1} \mathrm{H}-\mathrm{NMR}(200 \mathrm{MHz}$, DMSO$\left.d_{6}\right) \delta: 4.88$ (br, $\mathrm{D}_{2} \mathrm{O}$ exchangeable, $\left.1 \mathrm{H}, \mathrm{CH}_{2}-\mathrm{NH}\right), 5.85(\mathrm{~s}$, $\left.2 \mathrm{H}, \underline{\mathrm{CH}}_{2}-\mathrm{NH}\right), 7.04-7.25$ ( $\mathrm{m}, 3 \mathrm{H}, \mathrm{H} 5$ and $\mathrm{H} 6$ benzimidazole, $\mathrm{H} 4$ ' furyl), 7.52-7.61 (m, 3H, $\mathrm{H} 4$ and $\mathrm{H} 7$ benzimidazole, H3' furyl), 7.79-7.83 (m, 1H, H5' furyl), 8.22 (br, 1H, NH pyrimidine, $\mathrm{D}_{2} \mathrm{O}$ exchangeable), $12.54(\mathrm{br}, 2 \mathrm{H}, \mathrm{COOH}$ and $\mathrm{NH}$ benzimidazole, $\mathrm{D}_{2} \mathrm{O}$ exchangeable); IR $\left(\mathrm{KBr}, \mathrm{cm}^{-1}\right) 3300(\mathrm{NH}$ benzimidazole, $\mathrm{NH}-\mathrm{CH}_{2}$ ), 3159 (br, $\mathrm{H}$ bonded $\mathrm{OH}$ carboxylic, NH pyrimidine), 3050 (CH arom.), 2929 (CH aliph.), 1698 $(\mathrm{C}=\mathrm{O}$ carboxylic, amide), $1620(\mathrm{C}=\mathrm{N}$ benzimidazole $), 1558$, $1538(\mathrm{C}=\mathrm{N}$ pyrimidine, $\mathrm{C}=\mathrm{C})$. Anal. Calcd for $\mathrm{C}_{17} \mathrm{H}_{13} \mathrm{~N}_{5} \mathrm{O}_{4}: \mathrm{C}$, 58.12; H, 3.74, N ; 19.94. Found: C, 58.32; H, 3.52; N, 19.75.

Hepatitis C Virus (HCV) Viral RNA Replication Inhibitory Activity. Generation of HCV Replicon Cells The applied method was previously described by Lin et al. ${ }^{8)}$ Parental Huh-7 cells were cultured in Dulbecco's modified Eagle's medium (DMEM; JRH Biosciences, Lenexa, KS, U.S.A.) containing $10 \%$ heat-inactivated fetal bovine serum $(\triangle \mathrm{FBS}$; JRH Biosciences), $2 \mathrm{~mm}$ L-glutamine, and nonessential amino acids (JRH Biosciences). The cells were transfected with an in vitro-transcribed subgenomic $\mathrm{HCV}$ replicon RNA whose sequence was identical to that of the $\mathrm{I}_{377}$ neo/NS3-3'/wt replicon described by Lohmann et al. ${ }^{40)}$ Stable cells containing the self-replicating $\mathrm{HCV}$ replicon were selected and maintained in the presence of $250 \mu \mathrm{g}$ of G418 (Invitrogen, Carlsbad, CA, U.S.A.) per $\mathrm{mL}$ and were used for $\mathrm{HCV}$ replicon assays.

HCV Replicon Assay HCV replicon cells were plated in a 96-well plate at a density of $10^{4}$ cells per well in DMEM with $10 \% \triangle \mathrm{FBS}$. On the following day ( $c a$. 16h later), the culture medium was replaced with DMEM containing either no compound as a control or compounds serially diluted in the presence of $2 \% \triangle \mathrm{FBS}$ and $0.5 \%$ dimethyl sulfoxide (DMSO). After the cells were incubated with the compounds for $48 \mathrm{~h}$, the intracellular RNA was extracted with an RNeasy 96 kit (Qiagen, Valencia, CA, U.S.A.). The level of HCV RNA, both the positive strand and the negative strand, was determined by a real-time multiplex quantitative reverse transcription-polymerase chain reaction (RT-PCR) assay (the Taqman assay) with a pair of $\mathrm{HCV}$-specific primers (5'-CCA TGA ATC ACT CCC CTG TG-3' and 5'-CCG GTC GTC CTG GCA ATT C-3'), an HCV-specific probe (5',6-FAM-CCT GGA GGC TGC ACG ACA CTC A-TAMRA-3', where FAM is 6-carboxyfluorescein and TAMRA is 6-carboxytetramethylrhodamine), and an ABI Prism 7700 sequence detection system (Applied Biosystems, Foster City, CA, U.S.A.). In each experiment, each datum point represents the average of five replicates in cell culture. The $\mathrm{IC}_{50}$ was defined as the concentration of compound at which the HCV RNA level in the replicon cells was reduced by $50 \%$. To monitor any cytotoxic effect, the viabilities of the replicon cells following $48 \mathrm{~h}$ of treatment with compound were determined by using a tetrazolium compound (3-[4,5-dimethylthiazol-2-yl]-5-[3carboxymethoxyphenyl]-2-[4-sulfophenyl]-2H-tetrazolium, inner salt (MTS))-based assay (CellTiter 96 AQueous One Solution Cell Proliferation Assay; Promega, Madison, WI, 
U.S.A.). The $\mathrm{CC}_{50}$ was defined as the concentration of the compound at which cell viability was reduced by $50 \%$. The data obtained are presented in Table 1.

Docking. Generation of Structures and Exploration of the Conformational Space Structure building and refinement for $\mathbf{4 d}$ and $\mathbf{6 d}$ was accomplished using SYBYLX 1.1 molecular modeling software. ${ }^{41)}$ The obtained structures were used in this study and will be used in another 3D-QSAR study within our group.

First, we performed a grid search, using the Tripos force field $^{42)}$ with Gasteiger-Marsili charges ${ }^{43,44)}$ on the phenylpyrimidine fragment, iterating the bond between pyrimidine and phenyl in steps of $30^{\circ}$. Afterwards, we accomplished full conformational analyses of the entire molecule, while only the lowest energy conformer of phenylpyrimidine was retained from the previous calculation and kept fixed as an aggregate this time. The conformations for each compound obtained from these analyses were separated into 8 to 17 conformational families employing a hierarchical clustering algorithm.

The most reasonable low energy conformer was chosen from each of the obtained clusters. Finally, the two ligands were optimized with MOPAC using a semiempirical AM1 Hamiltonian $^{45,46)}$ to improve the molecular geometries and ensure better comparability of the ligand structures by providing structures based on identical levels of calculation.

Docking of Benzimidazole Inhibitors We chose the structure of NS3-4A serine protease bound to SCH446211 (PDB ID: 2FM2) as representative high resolution parent structure for docking $2.7 \AA$. Crystal structure was downloaded from the http://www.rcsb.org web site. All hydrogen atoms were added and the inhibitor and water molecules were removed. An active site of $5 \AA$ around the docked inhibitor was created. In all cases the specified size was sufficient to encompass the whole of the active site. To validate our docking procedure, the ligand was removed then docked back into the empty structure using genetic optimization for ligand docking (GOLD) software that uses the genetic algorithm (GA) to explore the full range of ligand conformational flexibility with partial flexibility of the protein. The algorithm's predictions were compared with the experimentally observed binding mode.

Our docking resulted in much closed conformation with the co-crystallised structure present in the protein data bank, which validates our docking procedure.

Gln41, Arg109, Ile132, Leu135, Lys136, Gly137, Phe154, Ala156, Ala157, and Cys159 were among the active site residues as identified from the known complexes from crystal structures.

We subsequently docked the two benzimidazole inhibitors into the NS3-4A serine protease structures using the covalent bond with the (Ser139) as a key requirement for a good solution.

The maximum number of generic algorithm runs was set to 20 for each compound. The default generic algorithm parameters were selected (100 population size, 5 number of islands, 100000 number of generic operations and 2 for the niche size). When the top three solutions attained RMSD values within $1.5 \AA$, GA docking was terminated.

The two scoring functions used, were the GoldScore fitness function and the ChemScore. ${ }^{47,48)}$ The GoldScore function is a molecular mechanics-like function. On the other hand, the
ChemScore function estimates the free energy of binding of the ligand to a protein.

Acknowledgment This study was supported by the "USEgypt Joint Science and Technology Board Fund" administered through the USDA (BIO9-002-015).

\section{References}

1) Memon M. I., Memon M. A., J. Viral Hepat., 9, 84-100 (2002).

2) Wasley A., Alter M. J., Semin. Liver Dis., 20, 1-16 (2000).

3) Manns M. P., McHutchison J. G., Gordon S. C., Rustgi V. K., Shiffman M., Reindollar R., Goodman Z. D., Koury K., Ling M.-H., Albrecht J. K., Lancet, 358, 958-965 (2001).

4) Fried M. W., Shiffman M. L., Reddy K. R., Smith C., Marinos G., Goncales F. L. Jr., Haussinger D., Diago M., Carosi G., Dhumeaux D., Craxi A., Lin A., Hoffman J., Yu J., N. Engl. J. Med., 347, 975-982 (2002).

5) Grakoui A., McCourt D. W., Wychowski C., Feinstone S. M., Rice C. M., J. Virol., 67, 2832-2843 (1993).

6) Kim J. L., Morgenstern K. A., Lin C., Fox T., Dwyer M. D., Landro J. A., Chambers S. P., Markland W. C., Lepre A., O'Malley E. T., Harbeson S. L., Rice C. M., Murcko M. A., Caron P. R., Thomson J. A., Cell, 87, 343-355 (1996).

7) Lin C., Thomson J. A., Rice C. M., J. Virol., 69, $4373-4380$ (1995).

8) Lin K., Kwong A. D., Lin C., Antimicrob. Agents Chemother., 48, 4784-4792 (2004).

9) Kolykhalov A. A., Mihalik K., Feinstone S. M., Rice C. M., J. Virol., 74, 2046-2051 (2000).

10) Perni R. B., Kwong A. D., Prog. Med. Chem., 39, 215-255 (2002).

11) De Francesco R., Tomei L., Altamura S., Summa V., Migliaccio G., Antiviral Res., 58, 1-16 (2003).

12) Grakoui A., McCourt D. W., Wychowski C., Feinstone S. M., Rice C. M., Proc. Natl. Acad. Sci. U.S.A., 90, 10583-10587 (1993).

13) Grakoui A., Wychowski C., Lin C., Feinstone S. M., Rice C. M., J. Virol., 67, 1385-1395 (1993).

14) Hijikata M., Mizushima H., Tanji Y., Komoda Y., Hirowatari Y., Akagi T., Kato N., Kimura K., Shimotohno K., Proc. Natl. Acad. Sci. U.S.A., 90, 10773-10777 (1993).

15) Bartenschlager R., Ahlborn-Laake L., Mous J., Jacobsen H., J. Virol., 67, 3835-3844 (1993).

16) Tomei L., Failla C., Santolini E., De Francesco R., La Monica N., J. Virol., 67, 4017-4026 (1993).

17) Tanji Y., Hijikata M., Satoh S., Kaneko T., Shimotohno K., J. Virol., 69, 1575-1581 (1995).

18) Love R. A., Parge H. E., Wickersham J. A., Hostomsky Z., Habuka N., Moomaw E. W., Adachi T., Hostomska Z., Cell, 87, 331-342 (1996).

19) Kim J. L., Morgenstern K. A., Lin C., Fox T., Dwyer M. D., Landro J. A., Chambers S. P., Markland W., Lepre C. A., O’Malley E. T., Harbeson S. L., Rice C. M., Murcko M. A., Caron P. R., Thomson J. A., Cell, 87, 343-355 (1996).

20) Cicero D. O., Barbato G., Koch U., Ingallinella P., Bianchi E., Nardi M. C., Steinkühler C., Cortese R., Matassa V. G., De Francesco R., Pessi A., Bazzo R., J. Mol. Biol., 289, 385-396 (1999).

21) Barbato G., Cicero D. O., Cordier F., Narjes F., Gerlach B., Sambucini S., Grzesiek S., Matassa V. G., De Francesco R., Bazzo R., EMBO J., 19, 1195-1206 (2000).

22) De Francesco R., Migliaccio G., Nature (London), 436, 953-960 (2005).

23) Gentile I., Borgia F., Buonomo A. R., Castaldo G., Borgia G., Curr. Med. Chem., 20, 3733-3742 (2013).

24) Tong X., Chase R., Skelton A., Chen T., Wright-Minogue J., Malcolm B. A., Antiviral Res., 70, 28-38 (2006).

25) Gentile I., Viola C., Borgia F., Castaldo G., Borgia G., Curr. Med. Chem., 16, 1115-1121 (2009).

26) Perni R. B., Almquist S. J., Byrn R. A., Chandorkar G., Chaturvedi 
P. R., Courtney L. F., Decker C. J., Dinehart K., Gates C. A., Harbeson L., Heiser A., Kalkeri G., Kolaczkowski E., Lin K., Luong Y. P., Rao B. G., Taylor W. P., Thomson J. A., Tung R. D., Wei Y., Kwong A. D., Lin C., Antimicrob. Agents Chemother., 50, 899-909 (2006).

27) Forestier N., Reesink H. W., Weegink C. J., McNair L., Kieffer T. L., Chu H. M., Purdy S., Jansen P. L., Zeuzem S., Hepatology, 46, 640-648 (2007).

28) Katz B. A., Clark J., Finer-Moore J., Jenkins T., Johnson C., Ross M., Luong C., Moore W., Stroud R., Nature (London), 391, 608-612 (1998).

29) Sperandio D., Gangloff A. R., Litvak J., Goldsmith R., Hataye J. M., Wang V. R., Shelton E. J., Elrod K., Janc J. W., Clark J. M., Rice K., Weinheimer S., Yeung K., Meanwell N. A., Hernandez D., Staab A. J., Venables B. L., Spencer J. R., Bioorg. Med. Chem. Lett., 12, 3129-3133 (2002).

30) Abdel-Mohsen H. T., Ragab F. A. F., Ramla M. M., El Diwani H. I., Eur. J. Med. Chem., 45, 2336-2344 (2010).

31) Skolnik B. H., Miller J. G., Day A. R., J. Am. Chem. Soc., 65 , 1854-1858 (1943).

32) Ding Y., Girardet J. L., Smith K. L., Larson G., Prigaro B., Wu J. Z., Yao N., Bioorg. Chem., 34, 26-38 (2006).

33) Kambe S., Saito K., Kishi H., Sakurai A., Midorikawa H., Synthesis, 1979, 287-289 (1979).

34) Ram V. J., Arch. Pharm. (Weinheim), 323, 895-899 (1990).

35) Cambridge Crystallographic Data Centre, "GOLD, Version 5.1.1.": 〈http://www.ccdc.cam.ac.uk/products/life_sciences/gold/s.
36) Jones G., Willett P., Glen R. C., J. Mol. Biol., 245, $43-53$ (1995).

37) Jones G., Willett P., Glen R. C., Leach A. R., Taylor R., J. Mol. Biol., 267, 727-748 (1997).

38) Yi M., Tong X., Skelton A., Chase R., Chen T., Prongay A., Bogen S. L., Saksena A. K., Njoroge F. G., Veselenak R. L., Pyles R. B., Bourne N., Malcolm B. A., Lemon S. M., J. Biol. Chem., 281, 8205-8215 (2006).

39) LaPlante S. R., Cameron D. R., Aubry N., Lefebvre S., Kukolj G., Maurice R., Thibeault D., Lamarre D., Llinàs-Brunet M., J. Biol. Chem., 274, 18618-18624 (1999).

40) Lohmann V., Körner F., Koch J. O., Herian U., Theilmann L., Bartenschlager R., Science, 285, 110-113 (1999).

41) SYBYL-X 1.1, Tripos International, 1699 South Hanley Rd., St. Louis, Missouri 63144, U.S.A.

42) Clark M., Cramer R. D., Van Opdenbosch N., J. Comput. Chem., 10, 982-1012 (1989).

43) Gasteiger J., Marsili M., Tetrahedron, 36, 3219-3228 (1980).

44) Hinze J., Jaffe H. H., J. Am. Chem. Soc., 84, 540-546 (1962).

45) Stewart J. J., J. Comput. Aided Mol. Des., 4, 1-103 (1990).

46) Stewart J. J. P., Frank J., MOPAC, Seiler Bokstav Research Laboratory, United Air Force Academy, CO 80840, U.S.A.

47) Verdonk M. L., Berdini V., Hartshorn M. J., Mooij W. T., Murray C. W., Taylor R. D., Watson P., J. Chem. Inf. Comput. Sci., 44, 793-806 (2004).

48) Jones G., Wilett P., Glen R. C., Leach A. R., Taylor R., J. Mol. Biol., 267, 727-748 (1997). 